Article

\title{
Entropy Generation Analysis of Open Parallel Microchannels Embedded Within a Permeable Continuous Moving Surface: Application to Magnetohydrodynamics (MHD)
}

\author{
Mohammad H. Yazdi ${ }^{1,2, *}$, Shahrir Abdullah ${ }^{1}$, Ishak Hashim ${ }^{3}$ and Kamaruzzaman Sopian ${ }^{2}$ \\ 1 Department of Mechanical and Materials Engineering, Faculty of Engineering and Built \\ Environment, Universiti Kebangsaan Malaysia, 43600 UKM, Bangi, Selangor, Malaysia; \\ E-Mail: shahrir@ukm.my \\ 2 Solar Energy Research Institute (SERI), Universiti Kebangsaan Malaysia, 43600 UKM, Bangi, \\ Selangor, Malaysia; E-Mail: ksopian@vlsi.eng.ukm.my \\ 3 School of Mathematical Sciences, Faculty of Science and Technology, Universiti Kebangsaan \\ Malaysia, 43600 UKM, Bangi, Selangor, Malaysia; E-Mail: ishak_h@ukm.my \\ * Author to whom correspondence should be addressed; \\ E-Mail: mohammadhossein.yazdi@gmail.com; Tel.: +603-8921-4596; Fax: +603-8921-4593.
}

Received: 11 October 2011; in revised form: 18 November 2011 / Accepted: 1 December 2011 / Published: 27 December 2011

\begin{abstract}
This paper presents a new design of open parallel microchannels embedded within a permeable continuous moving surface due to reduction of exergy losses in magnetohydrodynamic (MHD) flow at a prescribed surface temperature (PST). The entropy generation number is formulated by an integral of the local rate of entropy generation along the width of the surface based on an equal number of microchannels and no-slip gaps interspersed between those microchannels. The velocity, the temperature, the velocity gradient and the temperature gradient adjacent to the wall are substituted into this equation resulting from the momentum and energy equations obtained numerically by an explicit Runge-Kutta $(4,5)$ formula, the Dormand-Prince pair and shooting method. The entropy generation number, as well as the Bejan number, for various values of the involved parameters of the problem are also presented and discussed in detail.
\end{abstract}

Keywords: MHD; Joule heating; viscous dissipation; suction/injection; Entropy Based Surface Micro-Profiling (EBSM); embedded open parallel microchannels 


\section{Introduction}

The magnetohydrodynamic (MHD) flow and heat transfer in the presence of "slip" is an important topic in many engineering branches, especially in field of microelectromechanical systems (MEMS), such as micro MHD pumps [1], rapid mixing of biological fluids in biological processes [2,3], biological transportation, and drug delivery [4,5]. The magnetic field applied by a generating Lorenz force can control the electrically conducting fluid flow in a mixing process. However, as most of the applications of the biological transportation via an applied magnetic field are in the micro/nano systems [6-8], it is necessary to consider the influence of the velocity slip at the boundaries. Permeability is another effect that can act as transpiration of the boundaries in a micro system, which is an important aspect of micromixing of biological samples. In this process, suction is exerted in order to remove reactants, whereas injection is applied to add reactants in the process [2]. Therefore, many researchers have studied the boundary layer problems in the presence of "slip" [9-23]. Recently, Yazdi et al. [24] have investigated MHD liquid flow over nonlinear permeable stretching surface in the presence of the slip boundary condition and high-order chemical reactions.

Most of the methods developed for transporting particles and cells - such as pressure-driven flow, electrokinetics and electroosmosis methods - are usually applicable only for closed microchannels, with transportation in open microfluidic systems rarely being reported. Recently, Wu et al. [25] have evaluated a new method of transportation for particles, cells, and other microorganisms by rectified ac electro-osmotic flows in open microchannels. Their experimental study demonstrates that both driving electric field and gate potential can increase the particle velocity efficiently. Thus, the authors suggest using open microchannels instead of usual closed microchannels, since the former are open to the ambient air at the top, which can provide advantages, such as maintaining the physiological conditions for normal cell growth and introducing accurate amounts of chemical and biological materials [25]. Consequently, the need to combine MHD flow with open microchannels led to a new design of fluid transportation in micro systems.

The entropy based surface micro-profiling (EBSM) technique was developed for the first time by Naterer [26], who proposed surface microprofiling to reduce energy dissipation in convective heat transfer. This method includes local slip-flow conditions within the embedded open microchannels and thus tends to drag reduction and lower exergy losses along the surface. Naterer's results [27] imply that embedded surface microchannels can successfully reduce loss of available energy in convective heat transfer problems of viscous gas flow over a flat surface. In another study, Naterer [28] developed this technique to converging surface microchannels for minimized friction and thermal irreversibilities. The results of this work suggest that the embedded converging surface microchannels have the potential to reduce entropy generation in boundary layer flow with convective heat transfer. Naterer's results were obtained for gas-flow case based on EBSM to optimize open microchannels. However, in a subsequent work, Naterer et al. [29] have applied this method to the special application of aircraft intake de-icing, thus developing a new surface microprofiling technique for reducing exergy losses and controlling near-wall flow processes, particularly for anti-icing of a helicopter engine bay surface. Similarly, Yazdi et al. [30] have studied liquid fluid flow past embedded open parallel microchannels within the surface using EBSM. They show that EBSM can successfully reduce exergy losses in the liquid-flow problem. 
There have been many theoretical models developed specifically for entropy generation analysis of MHD boundary layer flow [31-37]. However, to the best of our knowledge, no investigation has been made yet to not only analyze the entropy generation of the slip MHD flow and heat transfer over permeable continuous moving surface but also to reach a new design of MHD flow over embedded surface microchannels. Therefore, the objective of this study is to reduce exergy losses of an electrically conducting fluid flow based on open parallel microchannels embedded within permeable continuous moving surface in the presence of applied magnetic field.

\section{Problem Formulation}

The flow configuration is illustrated in Figure 1. The 2-D, steady, laminar electrically conducting fluid flow over permeable continuous moving surface with embedded open parallel microchannels in the presence of applied magnetic field is considered. It is assumed that the width of the surface consists of a specific number of open microchannels and the base sections $\left(m^{\prime}\right)$, each of which has its own width. Moreover, a no-slip condition is applied between open microchannels, whereas a slip condition is applied to the open parallel microchannels. Thus, this arrangement requires simultaneous modeling of both slip-flow and no-slip conditions at the wall.

Figure 1. Schematic diagram of open parallel microchannels embedded within a surface.

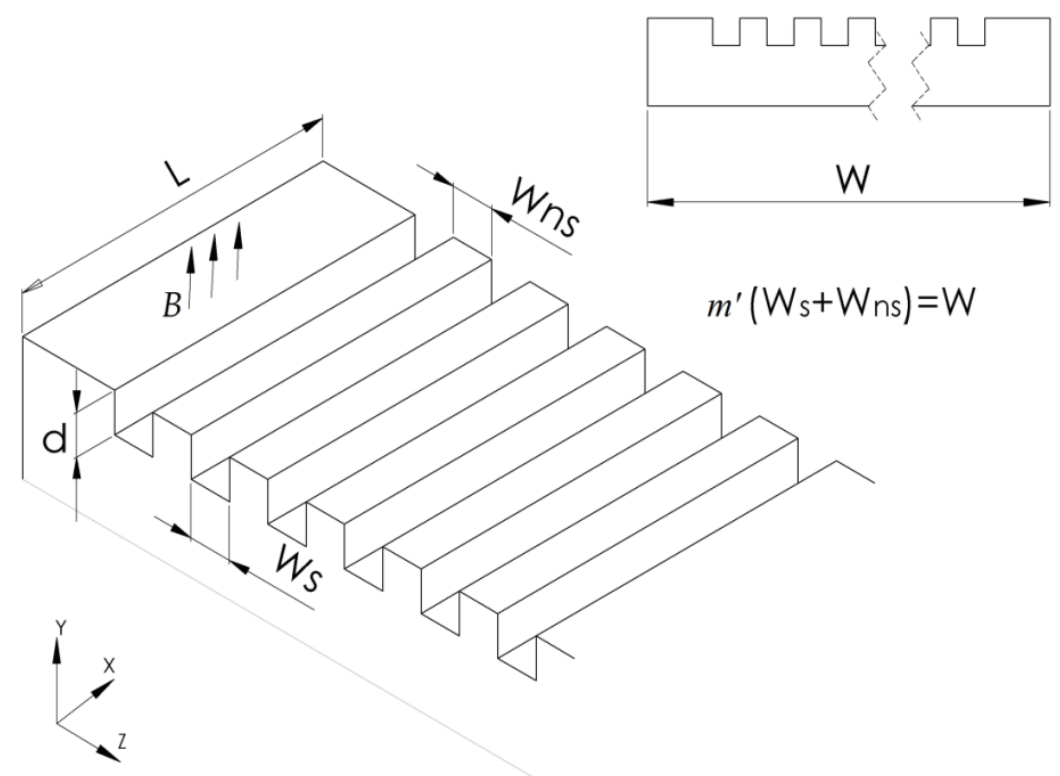

The fluid is a continuum, incompressible and Newtonian. In addition, as in an electrical insulator, the flow of electric current would give rise to the induced magnetic field, in this work, we have taken the fluid to be electrically conducting. Consequently, only the applied magnetic field plays a role and gives rise to the magnetic force [38]. Thus, the magnetic Reynolds number is assumed small and the induced magnetic field is neglected. We consider a transverse magnetic field with strength $B(x)$ which is applied in the vertical direction, given by the special form:

$$
B(x)=B_{0} x^{\frac{n-1}{2}}, B_{0} \neq 0
$$


The $x$-coordinate is determined along the surface, whilst the $z$ - and $y$-coordinates are measured perpendicular to the $x$ direction. Both viscous dissipation and Joule heating terms are considered in the energy equation. The corresponding velocity components in the $x$ and $y$ directions are $u$ and $v$, respectively. The velocity of the continuous moving surface is given by:

$$
u_{w}(x)=u_{0} x^{n}
$$

where $u_{0}$ is a constant rate parameter of the surface velocity and $n$ is a power index referring to the surface velocity parameter. The surface is at prescribed surface temperature (PST), $T_{w}$ given as:

$$
y=0, \quad T=T_{w}\left(=T_{\infty}+A x^{k^{\prime}}\right)
$$

where $A$ is a constant and $k^{\prime}$ is the surface temperature parameter at the prescribed surface temperature (PST) boundary condition. The steady two-dimensional MHD boundary layer equations for this problem, using the standard notation [24], are:

$$
\begin{gathered}
\frac{\partial u}{\partial x}+\frac{\partial v}{\partial y}=0 \\
u \frac{\partial u}{\partial x}+v \frac{\partial u}{\partial y}=v_{\infty} \frac{\partial^{2} u}{\partial y^{2}}-\frac{\sigma B^{2} u}{\rho} \\
u \frac{\partial T}{\partial x}+v \frac{\partial T}{\partial y}=\alpha \frac{\partial^{2} T}{\partial y^{2}}+\frac{v_{\infty}}{c_{p}}\left(\frac{\partial u}{\partial y}\right)^{2}+\frac{\sigma B^{2}}{\rho c_{p}} u^{2}
\end{gathered}
$$

The last two terms in the above energy Equation (6) are viscous dissipation and Joule heating effects, with the latter already incorporated in the previous work done by Yazdi et al. [24]. The associated boundary conditions are:

$$
\begin{gathered}
y=0 \Rightarrow u=u_{w}+u_{s}, v= \pm v_{w}, \quad, T=T_{w}\left(=T_{\infty}+A x^{k^{\prime}}\right) \\
y \rightarrow \infty \Rightarrow u=0, T=T_{\infty}
\end{gathered}
$$

where $\rho$ is the fluid density, $\alpha$ is thermal diffusivity, $\sigma$ is the electrical conductivity of the fluid, $v_{w}$ is the suction/ injection and $u_{s}$ is the velocity slip, assumed to be proportional to the local wall shear stress as follows:

$$
u_{s}=\left.l \frac{\partial u}{\partial y}\right|_{w}
$$

where $l$ is slip length, which is for Newtonian fluids usually expressed as a direct proportionality between the slip velocity and the shear rate at a wall. The slip length is defined as an extrapolated distance relative to the wall where the tangential velocity component vanishes $[39,40]$. As the no-slip boundary condition is only valid if the fluid flow adjacent to the wall is in thermodynamic equilibrium, high frequency of collisions between the fluid and the solid wall is required. However, as in small-scale systems, the collision frequency is typically not high enough to guarantee thermodynamic equilibrium, a certain degree of tangential velocity slip must be allowed [41]. To design a micropatterned surface in the presence of applied magnetic field, this slip boundary condition should 
be considered inside the open microchannels. Empirical evidence suggests that, for water flowing through a microchannel, the surface of which is coated with a $2.3 \mathrm{~nm}$ thick monolayer of hydrophobic octadecyltrichlorosilane, an apparent velocity slip is measured just above the solid surface. This velocity is approximately $10 \%$ of the free-stream velocity and yields a slip length of approximately $1 \mathrm{~mm}$ [42]. Thus, the slip boundary condition should be considered at the open parallel microchannels. Consequently, the fundamental equations of the boundary layer are transformed to ordinary differential ones that are locally valid. Thus, the mathematical analysis of the problem can be simplified by introducing the following dimensionless coordinates [24]:

$$
\begin{gathered}
f^{\prime}(\eta)=\frac{u}{u_{w}}=\frac{u}{u_{0} x^{n}} \quad, \quad \eta=y \sqrt{\frac{u_{0}(n+1)}{2 v_{\infty}}} x^{\frac{n-1}{2}}, \quad \theta(\eta)=\frac{T-T_{\infty}}{T_{w}-T_{\infty}} \\
v=-\sqrt{\frac{u_{0} v_{\infty}(n+1)}{2}} x^{\frac{n-1}{2}}\left(f+\frac{n-1}{n+1} \eta f^{\prime}\right)
\end{gathered}
$$

Here, it is useful to introduce a slip coefficient using similarity variables:

$$
f^{\prime}(0)=1+K f^{\prime \prime}(0)
$$

where $K$ is the slip coefficient defined for liquids by:

$$
K=l \sqrt{\frac{u_{w}(n+1)}{2 x v_{\infty}}}
$$

The fundamental partial differential Equations (5) and (6) are transformed to ordinary differential equations by substituting relevant variables (9) into Equations (5) and (6) as follows:

$$
\begin{gathered}
f^{\prime \prime \prime}+f f^{\prime \prime}-\left(\frac{2 M^{2}}{n+1}\right) f^{\prime}-\left(\frac{2 n}{n+1}\right)\left(f^{\prime}\right)^{2}=0 \\
\theta^{\prime \prime}+\operatorname{Pr} \theta^{\prime} f-\left(\frac{2 k^{\prime}}{n+1}\right) \operatorname{Pr} f^{\prime} \theta+\operatorname{Ec} x^{2 n-k^{\prime}} \operatorname{Pr}\left[\left(f^{\prime \prime}\right)^{2}+\left(\frac{2 M^{2}}{n+1}\right) f^{\prime 2}\right]=0
\end{gathered}
$$

For these equations, the associated boundary conditions are:

$$
\eta=0 \Rightarrow\left\{\begin{array}{l}
f^{\prime}(0)=1+K f^{\prime \prime}(0) \\
f(0)=f_{w} \\
\theta(0)=1
\end{array} \quad, \quad \eta \rightarrow \infty \Rightarrow\left\{\begin{array}{l}
f^{\prime}(\infty)=0 \\
\theta(\infty)=0
\end{array}\right.\right.
$$

where $f_{w}, \operatorname{Pr}, \mathrm{Ec}$, and $M$ show the suction/injection parameter, the Prandtl number, the Eckert number and the magnetic parameter respectively:

$$
f_{w}=\frac{-v_{w}}{\sqrt{\frac{u_{w} v_{\infty}(n+1)}{2 x}}}, \quad M^{2}=\frac{\sigma B_{0}{ }^{2}}{\rho u_{0}}, \quad \mathrm{Ec}=\frac{u_{0}{ }^{2}}{A c_{p}} \quad, \quad \operatorname{Pr}=\frac{v_{\infty}}{\alpha}
$$

where $f_{w}<0$ for mass injection and $f_{w}>0$ in the presence of the suction along the surface. Based on the previous work [24], $f_{w p}$ and $K_{p}$ are introduced as suction/injection and slip coefficient, respectively, based on $P_{n x}$, which are fully independent from $x$ and $n$ : 


$$
\begin{gathered}
f_{w}=\frac{-v_{w}}{\sqrt{\frac{u_{w} v_{\infty}(n+1)}{2 x}}}=\frac{-v_{w}}{\sqrt{u_{0} v_{\infty} P_{n x}}}=\frac{f_{w p}}{\sqrt{P_{n x}}} \\
K=l \sqrt{\frac{u_{w}(n+1)}{2 x v_{\infty}}}=l \sqrt{\frac{u_{0} P_{n x}}{v_{\infty}}}=K_{p} \sqrt{P_{n x}}
\end{gathered}
$$

where $P_{n x}$ is defined as:

$$
P_{n x}=\frac{x^{n-1}(n+1)}{2}
$$

The one-way coupled Equations (12) and (13) are solved numerically by using the explicit Runge-Kutta $(4,5)$ formula, the Dormand-Prince pair and shooting method, subject to the boundary conditions (14). The results of the numerical solutions to the problem are subsequently substituted into the entropy generation analysis. It is shown that the wall shear stress and the local Nusselt number exhibit a dependence on the involved parameters of the problem as follows:

$$
\begin{aligned}
& \tau_{w}=\mu\left|\frac{\partial u}{\partial y}\right|_{y=0}=\mu u_{0} \sqrt{\frac{u_{0}(n+1)}{2 v_{\infty}} x^{\frac{3 n-1}{2}}\left|f^{\prime \prime}(0)\right|} \\
& N u_{x}=\frac{-\left.x \frac{\partial T}{\partial y}\right|_{y=0}}{T_{w}-T_{\infty}}=\left|\theta^{\prime}(0)\right| \sqrt{\left(\frac{u_{0}(n+1)}{2 v_{\infty}}\right)} x^{\frac{n+1}{2}}
\end{aligned}
$$

\section{Entropy Generation Analysis}

Entropy generation related to the MHD flow over a permeable continuous moving surface with embedded open microchannels at prescribed surface temperature (PST) is considered. Heat transfer $\left(S_{T}{ }^{\prime \prime}\right)$, friction $\left(S_{F}{ }^{\prime \prime}\right)$, and magnetic irreversibilities $\left(S_{M}{ }^{\prime \prime}\right)$ are included within the local volumetric rate of entropy generation. The rate of entropy generation will be obtained based on the previous solutions of the boundary layer for fluid velocity and temperature. According to Woods [43] and Aïboud [32], the local volumetric rate of entropy generation in the presence of a magnetic field is given by:

$$
\begin{aligned}
& S_{g}^{\prime \prime \prime}=\frac{k_{\infty}}{T_{\infty}}\left[\left(\frac{\partial T}{\partial x}\right)^{2}+\left(\frac{\partial T}{\partial y}\right)^{2}\right]+\frac{\mu}{T_{\infty}}\left(\frac{\partial u}{\partial y}\right)^{2}+\frac{\sigma B^{2}}{T_{\infty}} u^{2} \\
& =S_{T}^{\prime \prime \prime}+S_{F}^{\prime \prime \prime}+S_{M}^{\prime \prime \prime}
\end{aligned}
$$

In order to include the effect of the embedded open parallel microchannels within the surface, integration over the width of the surface is applied over the local rate of entropy generation adjacent to the wall. The cross-stream $(z)$ dependence arises from interspersed no-slip (subscript $n s$ ) and slip-flow (subscript $s$ ) solutions of the boundary layer equations. Therefore, the integration over the width of the surface from $0 \leq z \leq W$ consists of $m^{\prime}$ separate integrations over each microchannel surface width, $0 \leq z \leq W_{s}+2 d$, as well as the remaining no-slip portion of the plate, which is interspersed between 
these microchannels and covers a range of $0 \leq z \leq W-m^{\prime} W_{s}$ (see Figure 1). The previous correlations for the convection coefficient based on the velocity, temperature, velocity gradient and the temperature gradient adjacent to the wall are substituted into this equation. Thus, by performing the integrations, and assuming an equal number of microchannels and no-slip gaps interspersed between those microchannels, it can be shown that:

$$
S_{g}^{\prime \prime}=S_{T}^{\prime \prime}+S_{F}^{\prime \prime}+S_{M}^{\prime \prime}
$$

where:

$$
\begin{aligned}
& S_{T}^{\prime \prime}=\int_{0}^{m^{\prime}\left(W_{s}+2 d\right)} S_{T, s l i p}^{\prime \prime \prime} d z+\int_{0}^{W-m^{\prime} W_{s}} S_{T, n o-s l i p}^{\prime \prime \prime} d z= \\
& \int \frac{k_{\infty} A^{2} k^{\prime 2} x^{\left(2 k^{\prime}-2\right)}}{T_{\infty}^{2}} \theta_{s}^{2}(0)\left[m^{\prime}\left(W_{s}+2 d\right)\right] \\
& =\left\{\begin{array}{l}
+\frac{k_{\infty} A^{2} k^{\prime 2} x^{\left(2 k^{\prime}-2\right)}}{T_{\infty}^{2}} \theta_{n s}^{2}(0)\left[\left(W-m^{\prime} W_{s}\right)\right] \\
+\frac{k_{\infty} A^{2} x^{2 k^{\prime}} u_{0}(n+1) x^{n}}{2 v_{\infty} x T_{\infty}^{2}} \theta_{s}^{\prime 2}(0)\left[m^{\prime}\left(W_{s}+2 d\right)\right] \\
+\frac{k_{\infty} A^{2} x^{2 k^{\prime}} u_{0}(n+1) x^{n}}{2 v_{\infty} x T_{\infty}^{2}} \theta_{n s}^{\prime 2}(0)\left[\left(W-m^{\prime} W_{s}\right)\right]
\end{array}\right. \\
& S_{F}^{\prime \prime}=\int_{0}^{m^{\prime}\left(W_{s}+2 d\right)} S_{F, s l i p}^{\prime \prime \prime} d z+\int_{0}^{W-m^{\prime} W_{s}} S_{F, n o-s l i p}^{\prime \prime \prime} d z= \\
& =\left\{\begin{array}{l}
\frac{\mu_{\infty} u_{0}{ }^{2} x^{2 n}}{T_{\infty}}\left(\frac{u_{0}(n+1)}{2 v_{\infty}} x^{(n-1)}\right) f_{s}^{\prime \prime 2}(0)\left[m^{\prime}\left(W_{s}+2 d\right)\right] \\
+\frac{\mu_{\infty} u_{0}{ }^{2} x^{2 n}}{T_{\infty}}\left(\frac{u_{0}(n+1)}{2 v_{\infty}} x^{(n-1)}\right) f_{n s}^{\prime \prime 2}(0)\left[\left(W-m^{\prime} W_{s}\right)\right]
\end{array}\right. \\
& S_{M}^{\prime \prime}=\int_{0}^{m^{\prime}\left(W_{s}+2 d\right)} S_{M, s l i p}^{\prime \prime \prime} d z+\int_{0}^{W-m^{\prime} W_{s}} S_{M, n o-s l i p}^{\prime \prime \prime} d z= \\
& =\left\{\frac{\sigma u_{0}{ }^{2} B^{2} x^{2 n}}{T_{\infty}} f_{s}^{\prime 2}(0)\left[m^{\prime}\left(W_{s}+2 d\right)\right]+\frac{\sigma u_{0}{ }^{2} B^{2} x^{2 n}}{T_{\infty}} f_{n s}^{\prime 2}(0)\left[\left(W-m^{\prime} W_{s}\right)\right]\right.
\end{aligned}
$$

Clearly, the local rate of entropy generation adjacent to the wall has been obtained considering $\eta=0$. The local rate of entropy generation over microchannel surface in the presence of the magnetic field has thus incorporated three sources of entropy generation. The first term on the right-hand side of the equation is the local entropy generation due to heat transfer across a finite temperature difference, the second term is the local entropy generation due to fluid friction irreversibilities, and the third term 
is the irreversibilities due to the effect of the magnetic field. For completeness, the dimensionless local entropy generation rate is defined as a ratio of the local entropy generation rate and a characteristic entropy generation rate. Here, the characteristic entropy generation rate, based on the width of the surface, is defined as:

$$
S_{g_{0}}^{\prime \prime}=\frac{k_{\infty} \Delta T^{2} W}{L^{2} T_{\infty}^{2}}
$$

where $L$ is characteristic length scale. Consequently, the entropy generation number in terms of nondimensional geometrical parameters $(\lambda$ and $\zeta)$ is expressed as:

$$
N_{s}=\frac{S_{g}^{\prime \prime}}{S_{g_{0}}^{\prime \prime}}=\left\{\begin{array}{l}
\frac{k^{\prime 2}}{X^{2}} \theta_{s}^{2}(0)\left[m^{\prime} \lambda\right]+\frac{k^{\prime 2}}{X^{2}} \theta_{n s}^{2}(0)\left[1+2 m^{\prime} \varsigma-m^{\prime} \lambda\right]+\frac{\operatorname{Re}}{X^{2}}\left(\frac{n+1}{2}\right) \theta_{s}^{\prime 2}(0)\left[m^{\prime} \lambda\right] \\
+\frac{B r}{\Omega} \frac{\operatorname{Re}}{X^{2}}\left(\frac{n+1}{2}\right) f_{s}^{\prime \prime 2}(0)\left[1+2 m^{\prime} \varsigma-m^{\prime} \lambda\right] \\
+\frac{B r}{\Omega} \frac{M^{2} \operatorname{Re}}{X^{2}} f_{s}^{\prime 2}(0)\left[m^{\prime} \lambda\right]+\frac{B r}{\Omega} \frac{M^{2} \operatorname{Re}}{X^{2}} \frac{\operatorname{Re}}{X_{n s}^{2}}\left(\frac{n+1}{2}\right) f_{n s}^{\prime \prime 2}(0)\left[1+2 m^{\prime} \varsigma-m^{\prime} \lambda\right]
\end{array}\right.
$$

where $\mathrm{X}, \mathrm{Re}, \mathrm{Br}$ and $\Omega$ are, respectively, the non-dimensional surface length, the Reynolds number (based on the surface velocity), the Brinkman number (based on the surface velocity) and the dimensionless temperature difference. These parameters are given by the following relationships:

$$
B r=\frac{\mu_{\infty}\left(u_{0} x^{n}\right)^{2}}{k_{\infty} \Delta T}, \quad \operatorname{Re}=\frac{\left(u_{0} x^{n}\right) x}{v_{\infty}}, \quad X=\frac{x}{L}, \quad \Omega=\frac{\Delta T}{T_{\infty}}
$$

In addition, the above non-dimensional geometric parameters are defined as:

$$
\lambda=\frac{W_{s}+2 d}{W}, \quad \varsigma=\frac{d}{W}
$$

When the present equation of the entropy generation number (27) is compared with the entropy generation equation of Aïboud [32] when $m^{\prime}=0$ and $n=1$, it is evident that the former can be applied to a linear surface velocity problem $(n=1)$ without open parallel microchannels $\left(m^{\prime}=0\right)$. Moreover, a laminar boundary layer flow is also considered in this research. It should also be noted that, although the entropy generation number is a non-dimensional parameter, the surface length should be selected in order to ensure that the Reynolds number remains below the point of transition to turbulence at $\operatorname{Re}_{L}=5 \times 10^{5}$, as in contrast to the external convective heat transfer problem, the critical Reynolds number within an open/closed microchannel is 1800 [26,44]. This Reynolds number is based on the microchannel depth or hydraulic diameter (rather than plate length), which remains below the transition point of 1800 in this problem. In this study, the Bejan number is defined as the ratio of heat transfer irreversibility to total irreversibility due to heat transfer, fluid friction and magnetic field for the laminar MHD boundary layer flow. Mathematically, Bejan number is given as $[45,46]$ : 


$$
B e=\frac{\text { Heat trans fer irreversibility }}{\text { Entropy generation number }}=\frac{1}{1+\Phi}
$$

where $\Phi$ is the irreversibility distribution ratio which is defined as:

$$
\Phi=\frac{\text { Fluid friction irreversibility }+ \text { Magnetic field irreversibility }}{\text { Heat trans fer irreversib ility }}
$$

As the Bejan number ranges from 0 to 1 , it approaches zero when the entropy generation due to the combined effects of fluid friction and magnetic field is dominant. Similarly, $B e>0.5$ indicates that the irreversibility due to heat transfer dominates, with $B e=1$ as the limit at which the irreversibility is solely due to heat transfer [47]. Consequently, $0 \leq \Phi \leq 1$ indicates that the irreversibility is primarily due to the heat transfer irreversibility, whereas for $\Phi>1$ it is due to the sum of the fluid friction and magnetic field irreversibility.

\section{Results and Discussion}

Table 1 shows a comparison between the results of the present work and that of the previous works for the special case of no Joule heating effect, clearly indicating an excellent agreement. The combination effect of the slip coefficient $K_{p}$, the magnetic parameter $M$ and surface velocity parameter $n$, on the velocity gradient adjacent to the wall has been illustrated in Figure 2, which confirms the accuracy of our method by comparing the momentum equation results presented here with our previous work [24]. The results illustrate that increasing values of the magnetic parameter $M$ and $n$ tend to increase the wall shear stress, whereas the wall shear stress decreases in the presence of a high slip coefficient. Figure 3 illustrates the combined effect of the Joule heating, slip coefficient $K_{p}$ and the magnetic parameter $M$ on the heat transfer rate $\left|\theta^{\prime}(0)\right|$ when $f_{w p}=0.2, n=0.5, k^{\prime}=0.02, \operatorname{Pr}=5$, and $\mathrm{Ec}=0.1$. The results demonstrate that the heat transfer rate is decreased by Joule heating. Moreover, the Joule heating effect is much more significant for higher values of magnetic parameters. Finally, increasing both the slip coefficient and magnetic parameter reduces the heat transfer rate.

Table 1. Comparison of the wall temperature gradient $\left|\theta^{\prime}(0)\right|$ between the present results and those obtained previously for the special case without Joule heating effect.

\begin{tabular}{|c|c|c|c|c|c|c|c|}
\hline $\boldsymbol{n}$ & $\boldsymbol{k}^{\prime}$ & $\mathbf{P r}$ & $\begin{array}{c}\text { Ali [48] } \\
\mathbf{( 1 9 9 4 )}\end{array}$ & Ishak [49] (2009) & $\begin{array}{c}\text { Hayat [18] } \\
\mathbf{( 2 0 1 0 )}\end{array}$ & $\begin{array}{c}\text { Yazdi [24] } \\
\mathbf{( 2 0 1 1 )}\end{array}$ & Present Results \\
\hline & & & & $\begin{array}{c}\text { Finite difference } \\
\text { method }\end{array}$ & $\begin{array}{c}\text { Homotopy } \\
\text { analysis method }\end{array}$ & $\begin{array}{c}\text { The Dormand- } \\
\text { Prince pair and } \\
\text { shooting method }\end{array}$ & $\begin{array}{c}\text { The Dormand- } \\
\text { Prince pair and } \\
\text { shooting method }\end{array}$ \\
\hline & & & & & $\left|\theta^{\prime}(0)\right|$ & & $\left|\theta^{\prime}(0)\right|$ \\
\hline 1 & 0 & 0.72 & 0.4617 & & & 0.4631 & 0.4631 \\
\hline & & 1 & 0.5801 & & & 0.5818 & 0.5818 \\
\hline & & 3 & 1.1599 & & & 1.1647 & 1.1647 \\
\hline 1 & 1 & 0.72 & & 0.8086 & 0.8086 & 0.8086 & 0.8086 \\
\hline & & 1 & & 1 & 1 & 1 & 1 \\
\hline & & 3 & & 1.9237 & 1.9236 & 1.9238 & 1.9238 \\
\hline
\end{tabular}


Figure 2. The effects of the surface velocity parameter $n$, the slip coefficient $K_{p}$ and the magnetic parameter $M$ on $f^{\prime \prime}(0)$ when $f_{w p}=0.2$.

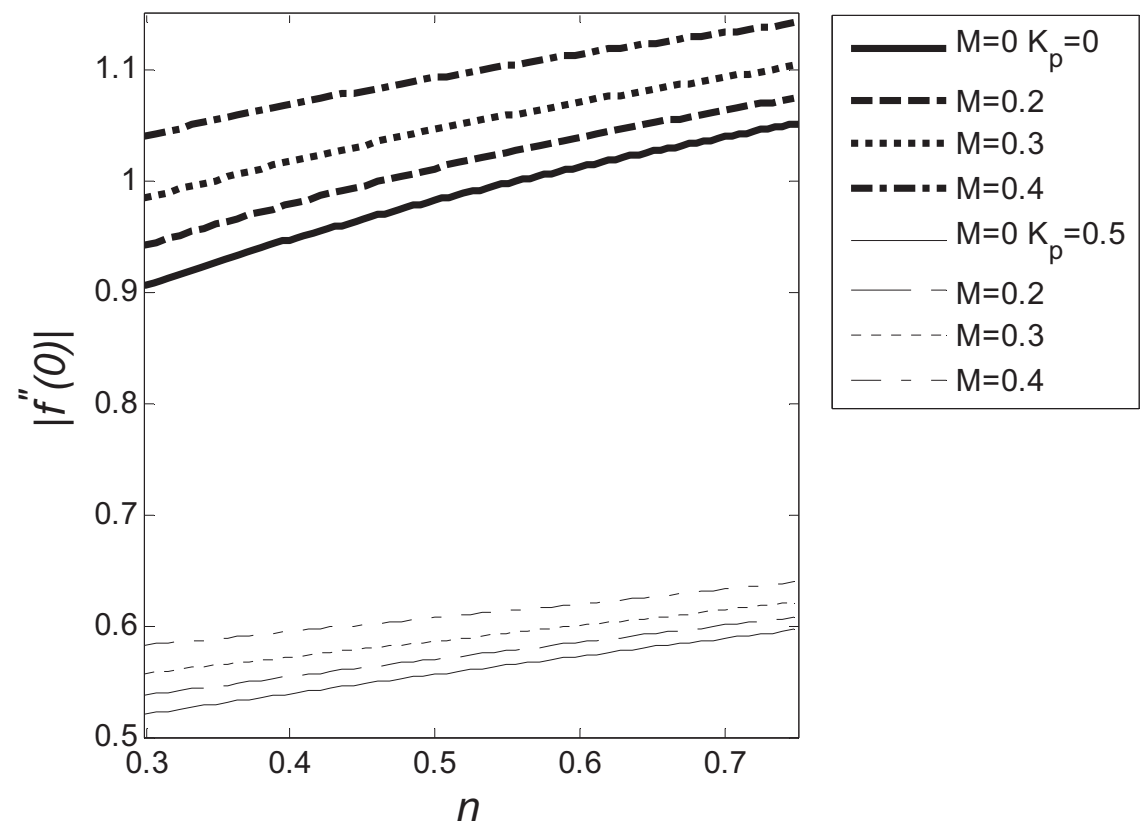

Figure 3. Variation in $\left|\theta^{\prime}(0)\right|$ as a function of $K_{p}$ for various values of $M$ when $f_{w p}=0.2$, $n=0.5, k^{\prime}=0.02, \operatorname{Pr}=5, \mathrm{Ec}=0.1$.

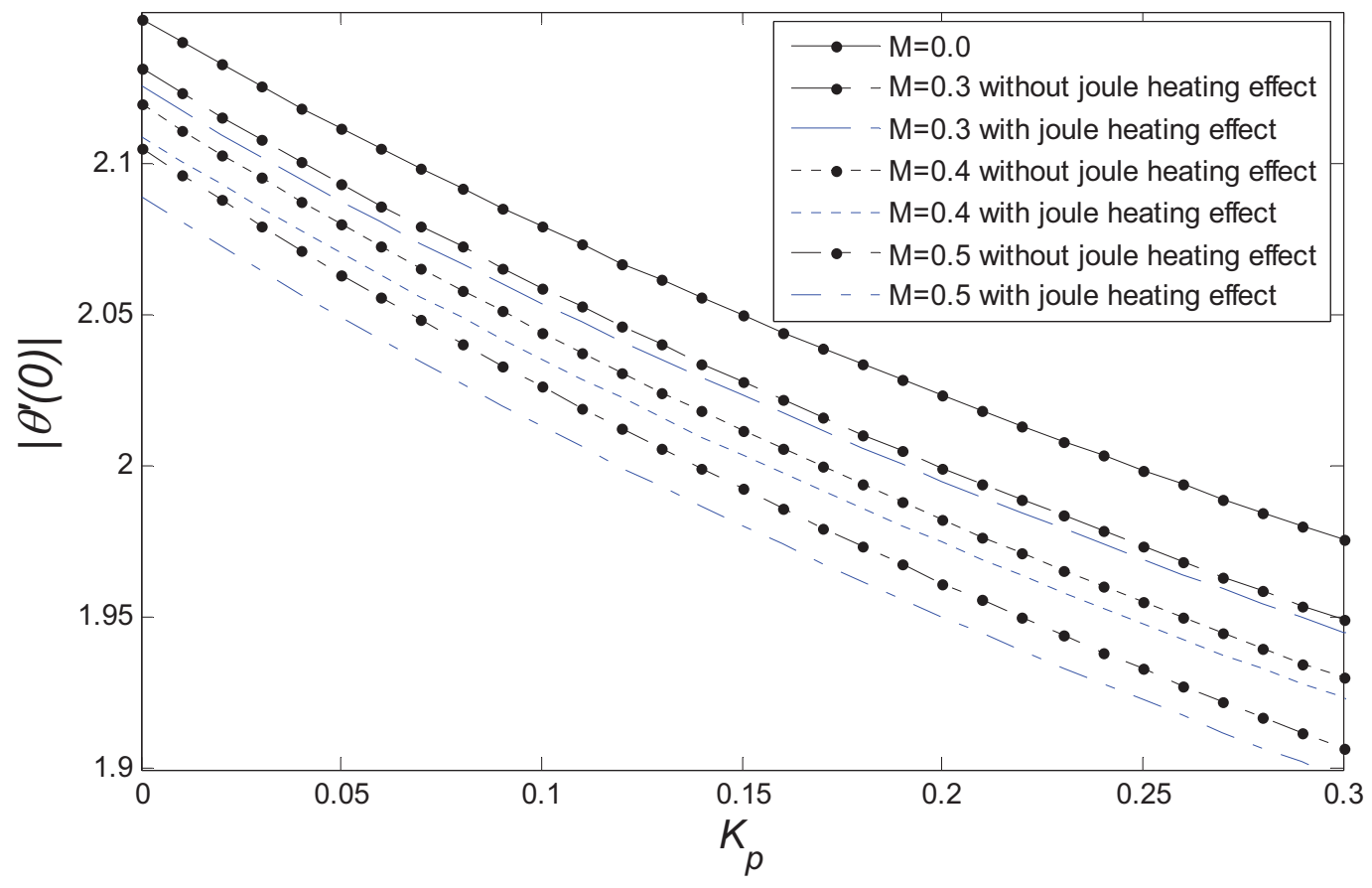

The following section presents the results for entropy generation analysis of MHD flow over open parallel microchannels embedded within a permeable continuous moving surface in the presence of Joule heating and viscous dissipation. The combination effect of the magnetic parameter and slip coefficient on the entropy generation number is illustrated in Figures 4 and 5 for different values of the dimensionless group parameter, $\operatorname{Br} \Omega^{-1}=0.1$ and $\mathrm{Br} \Omega^{-1}=1$, respectively. This design of embedded open parallel microchannels yields an interesting result with respect to reduction of the exergy losses 
along the surface structure. As mentioned before, the slip inside the open microchannels must be considered, particularly in cases where a hydrophobic microchannel surface exists. The current results demonstrate that the velocity slip at open parallel microchannels can decrease the entropy generation number adequately. However, the effect of the slip coefficient is not dependent on $\operatorname{Br} \Omega^{-1}$. Therefore, it can reduce both friction and heat transfer irreversibilities significantly due to its ability to decreasing both the wall shear stress and the heat transfer rate over a continuous moving surface. This result indicates that by means of open parallel microchannels embedded within the surface, the exergy losses decrease efficiently. As explained before, the entropy generation number is comprised of friction, heat transfer and magnetic irreversibilities. However, although the magnetic parameter reduces heat transfer irreversibilities by decreasing the heat transfer rate, it shows an opposite effect on both friction and magnetic irreversibilities. Thus, the magnetic parameter can decrease the total irreversibilities $\left(N_{s}\right)$ where the values of the heat transfer irreversibilities are much more significant compared to the friction irreversibilities, which occurs at low $\mathrm{Br} \Omega^{-1}$ (see Figure 4). The Brinkman number (Br) is a dimensionless number related to heat conduction from the surface to flowing viscous fluid. A reduction in the dimensionless group parameter $\mathrm{Br} \Omega^{-1}$ tends to simultaneously decrease both friction and magnetic irreversibilities. Consequently, magnetic parameter can decrease the entropy generation number at low $\operatorname{Br} \Omega^{-1}$. The effect of the magnetic parameter at high $\operatorname{Br} \Omega^{-1}$ is shown in Figure 5. It indicates that, although the magnetic parameter tends to decrease heat transfer irreversibilities, it is not sufficient to reduce the total irreversibilities along the surface structure. As a result, the remaining significant friction irreversibilities are still capable of increasing the total entropy generation along the open parallel microchannels embedded within the surface.

Figure 4. (a) Friction irreversibilities; (b) Heat transfer irreversibilities; (c) Magnetic irreversibilities; and (d) Entropy generation number as a function of $K_{p}$ for various values of magnetic parameter $M$ when $f_{w p}=0.2, n=0.5, \mathrm{X}=0.3, \mathrm{Re}=10, \operatorname{Pr}=5, \mathrm{Ec}=0.1$, $\mathrm{Br} \Omega^{-1}=0.1, k^{\prime}=0.02, m^{\prime}=100, \zeta=0.00001, \lambda=0.0001$.

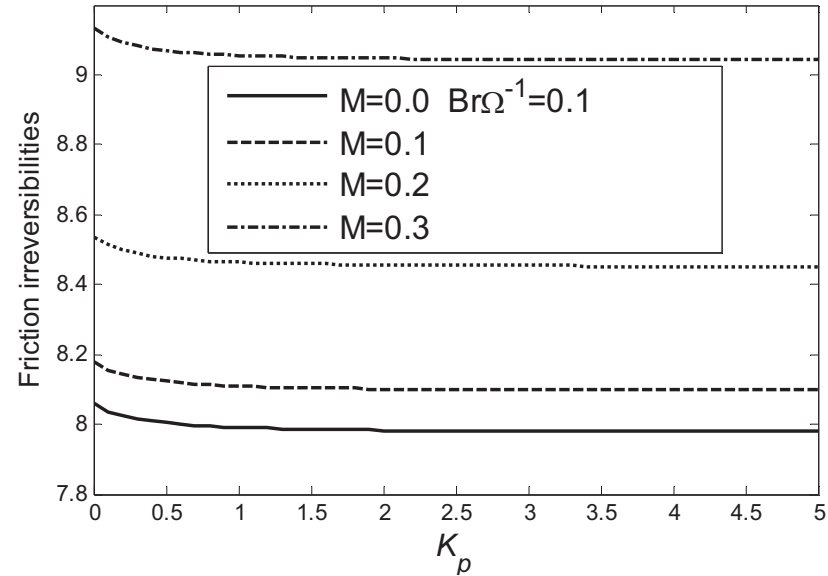

(a)

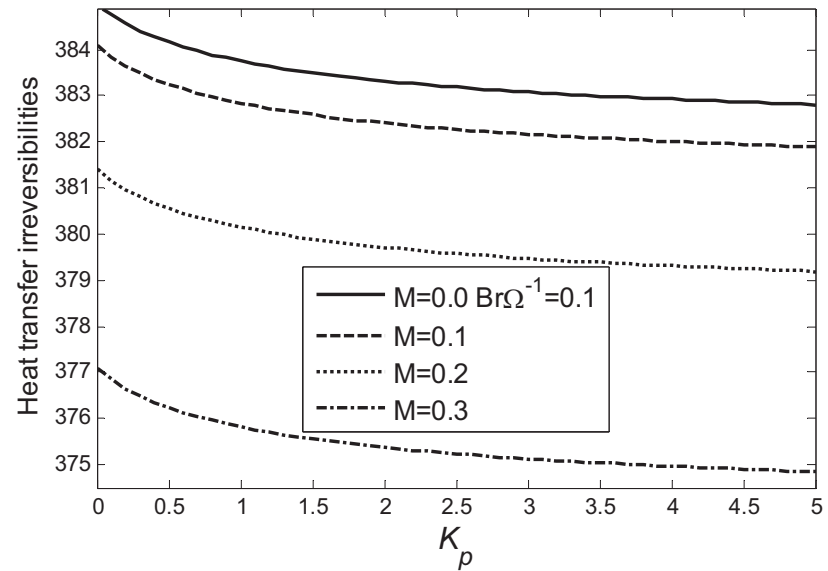

(b) 
Figure 4. Cont.

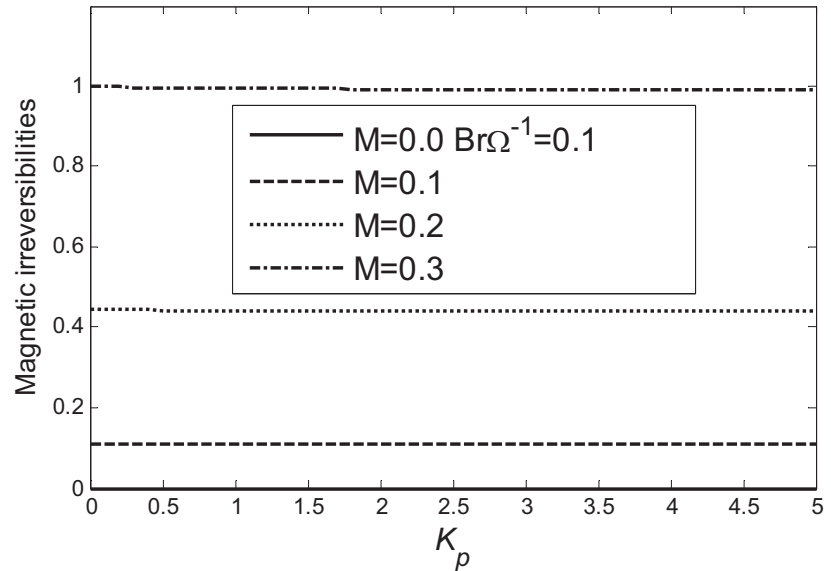

(c)

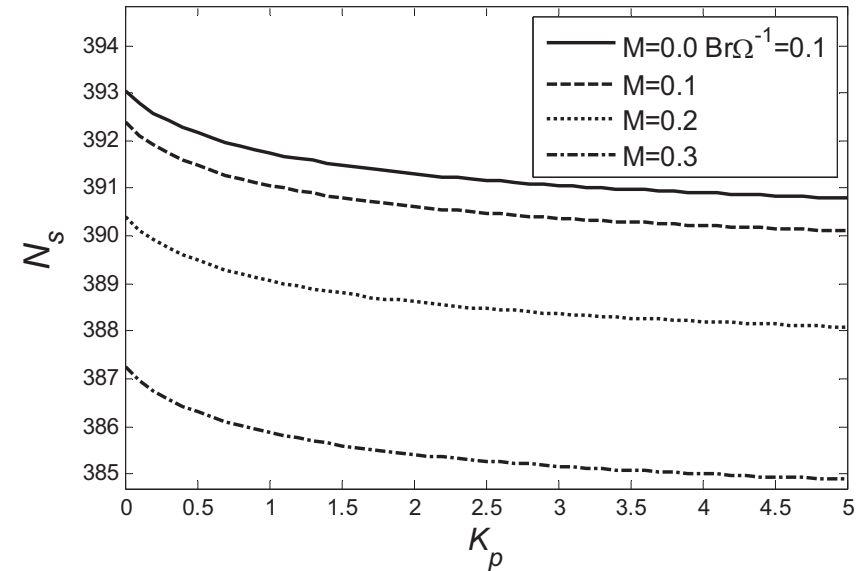

(d)

Figure 5. (a) Friction irreversibilities; (b) Heat transfer irreversibilities; (c) Magnetic irreversibilities; and (d) Entropy generation number as a function of $K_{p}$ for various values of magnetic parameter $M$ when $f_{w p}=0.2, n=0.5, \mathrm{X}=0.3, \operatorname{Re}=10, \operatorname{Pr}=5, \mathrm{Ec}=0.1$, $\mathrm{Br} \Omega^{-1}=1, k^{\prime}=0.02, m^{\prime}=100, \zeta=0.00001, \lambda=0.0001$.

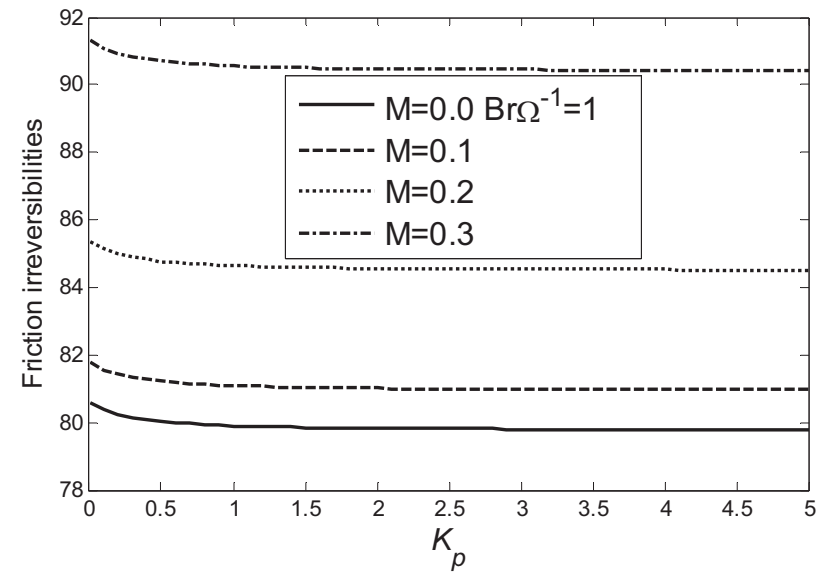

(a)

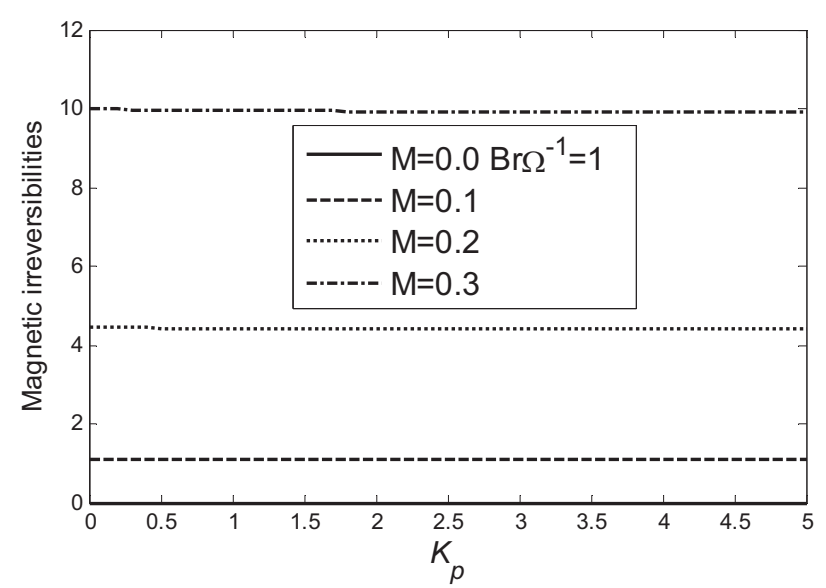

(c)

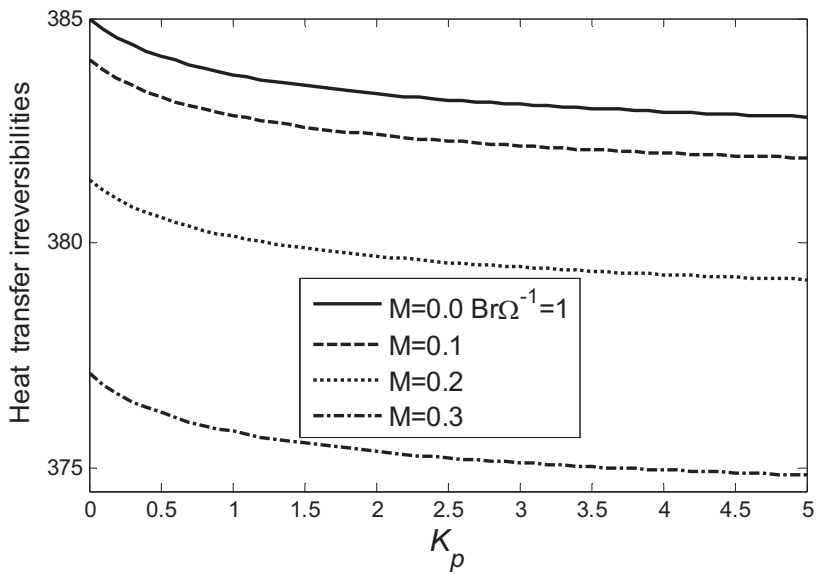

(b)

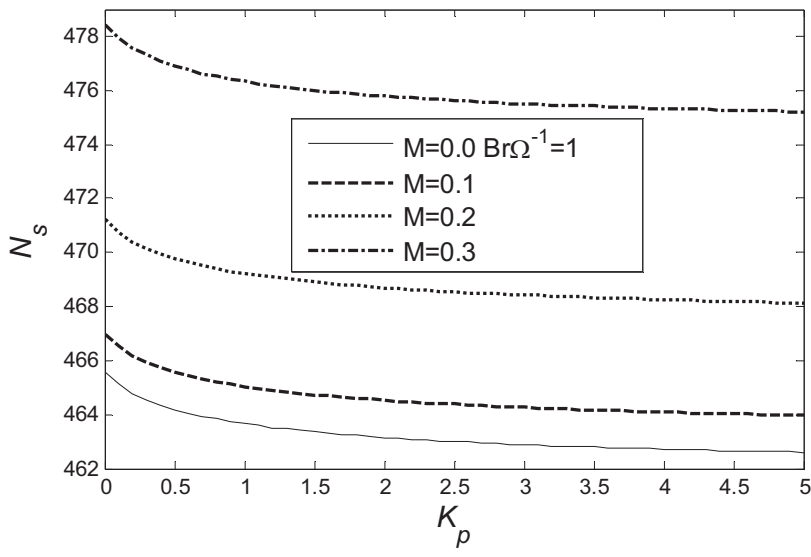

(d) 
The combined effect of the slip coefficient and magnetic parameter on the Bejan number is illustrated in Figure 6. In the following figures depicting Bejan number, it is observed that the Bejan number changes the trend, after reaching the maximum corresponding to a specific slip coefficient. It is interesting to note that, at the points to the left of the maximum, the slope of the tangent is positive, indicating that an increase in the $K_{p}$ tends to increase the Bejan number due to a reduction in the irreversibility distribution ratio $\Phi$. Similarly, at the points to the right, the slope is negative, i.e., higher $K_{p}$ values yield lower Bejan number. Further, it is noted that a decrease in both $M$ and $\operatorname{Br} \Omega^{-1}$ accompanies a rise in the Bejan number.

Figure 6. Bejan number as a function of $K_{p}$ for various values of magnetic parameter (a) $M=0.0, \mathrm{Br} \Omega^{-1}=0.1$; (b) $M=0.3, \mathrm{Br} \Omega^{-1}=0.1$; (c) $M=0.0, \mathrm{Br} \Omega^{-1}=1$; and (d) $M=0.3, \mathrm{Br} \Omega^{-1}=0.1$ when $f_{w p}=0.2, n=0.5, \mathrm{X}=0.3, \operatorname{Re}=10, \operatorname{Pr}=5, \mathrm{Ec}=0.1$, $k^{\prime}=0.02, m^{\prime}=100, \zeta=0.00001, \lambda=0.0001$.

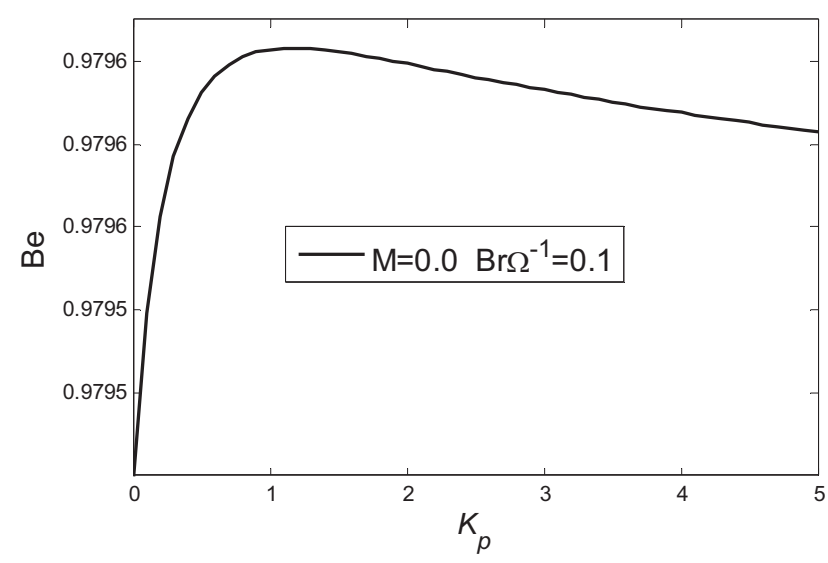

(a)

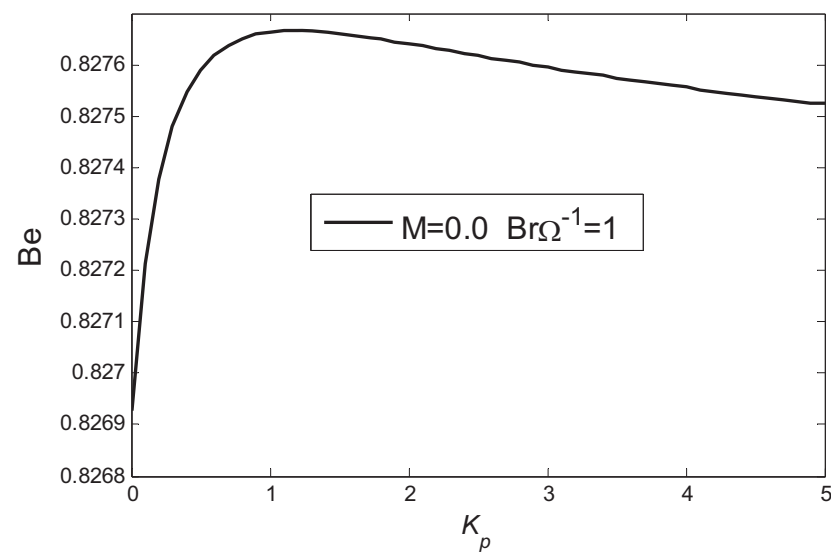

(c)

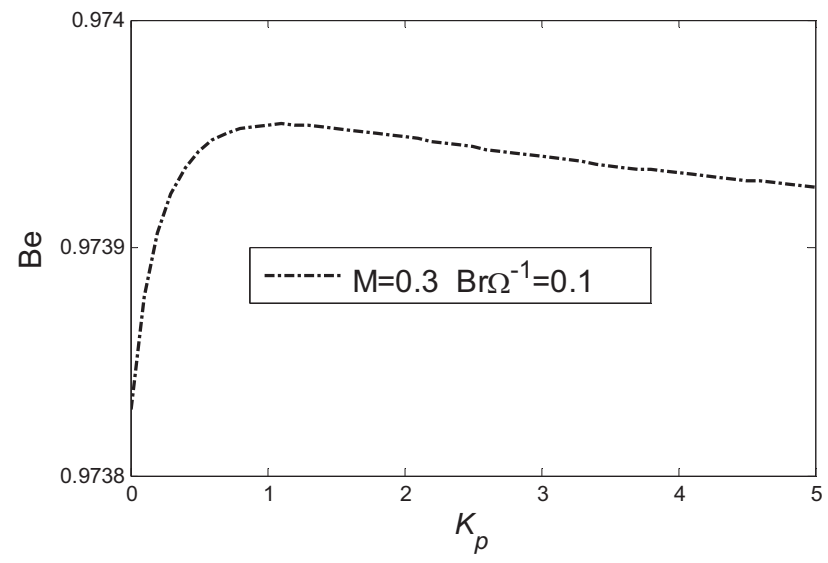

(b)

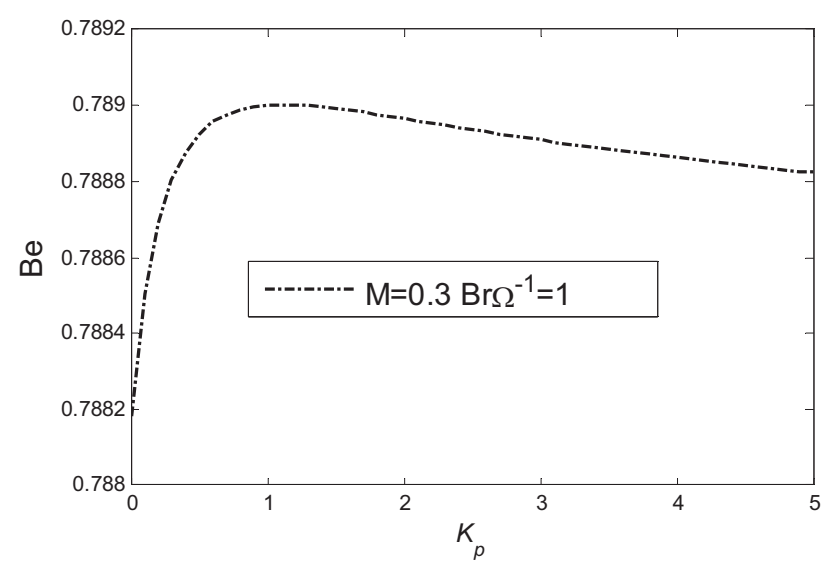

(d)

The effect of the group parameter $\mathrm{Br} \Omega^{-1}$ on the entropy generation number is shown in Figure 7 , where it is evident that an increase in the $\operatorname{Br} \Omega^{-1}$ tends to add more friction and magnetic irreversibilities to the entropy generation. Consequently, $N_{s}$ increases with the increase in $\operatorname{the} \operatorname{Br} \Omega^{-1}$. The effect of $\mathrm{Br} \Omega^{-1}$ on Bejan number is presented in Figure 8, where $\mathrm{Br} \Omega^{-1}$ appears just inside the friction and magnetic irreversibility Equations (27). Consequently, it has ability to control both friction and magnetic irreversibilities. It is observed that the heat transfer irreversibilities become much more 
dominant at low $\mathrm{Br} \Omega^{-1}$. The influence of the Reynolds number on the entropy generation number and Bejan number is illustrated in Figure 9. It is obvious that the increase in Reynolds number increases all three irreversibility parts of the entropy generation number $N_{s}$. In contrast, no considerable effect on the Bejan number is observed, the effect on all three parts of $N_{s}$ Equation (27) is similar.

Figure 7. Entropy generation number as a function of $K_{p}$ for various values of $\mathrm{Br} \Omega^{-1}$ when $f_{w p}=0.2, n=0.5, \mathrm{X}=0.3, \mathrm{Re}=10, \mathrm{Pr}=5, \mathrm{Ec}=0.1, M=0.1, k^{\prime}=0.2, m^{\prime}=100$, $\zeta=0.00001, \lambda=0.0001$.

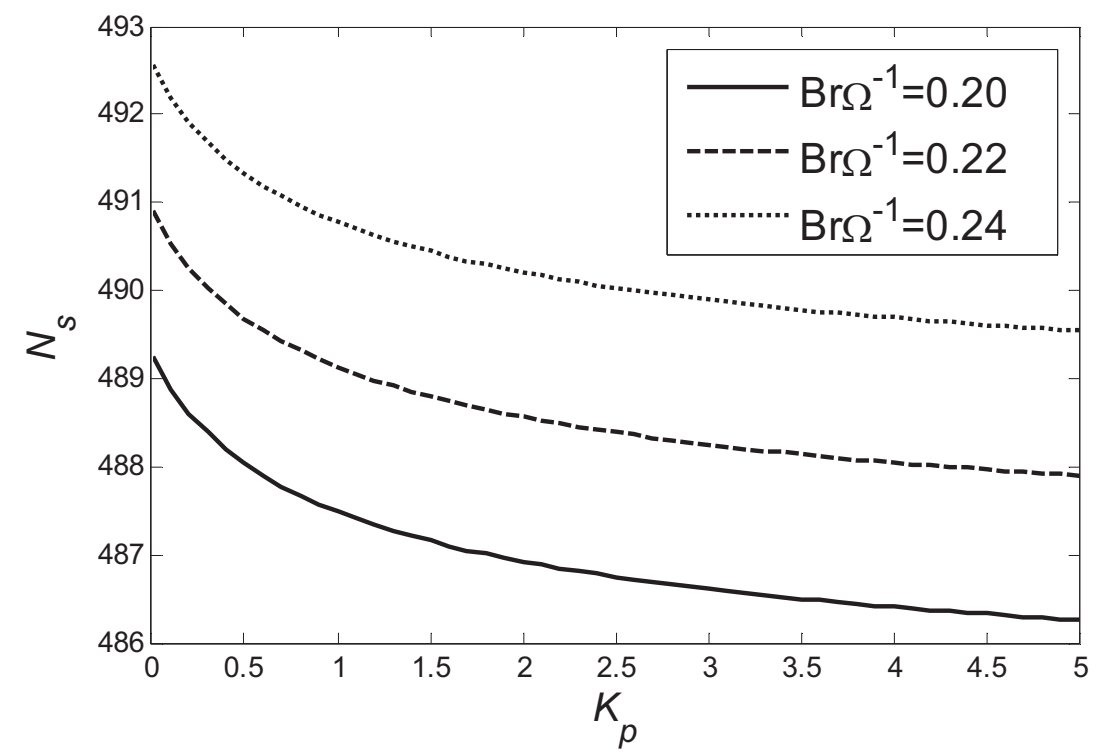

Figure 8. Bejan number as a function of $K_{p}$ for (a) $\mathrm{Br}^{-1}=0.20$ and (b) $\mathrm{Br}^{-1}=0.24$ when $f_{w p}=0.2, n=0.5, \mathrm{X}=0.3, \mathrm{Re}=10, \operatorname{Pr}=5, \mathrm{Ec}=0.1, M=0.1, k^{\prime}=0.2, m^{\prime}=100$, $\zeta=0.00001, \lambda=0.0001$.

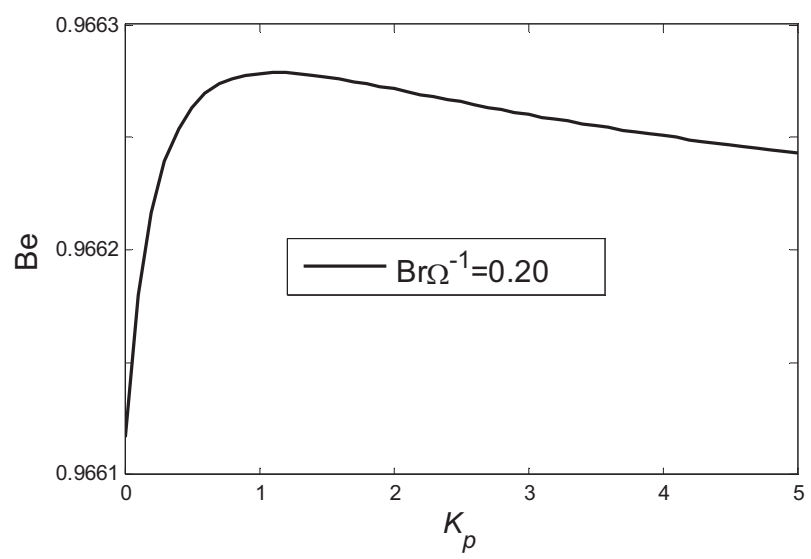

(a)

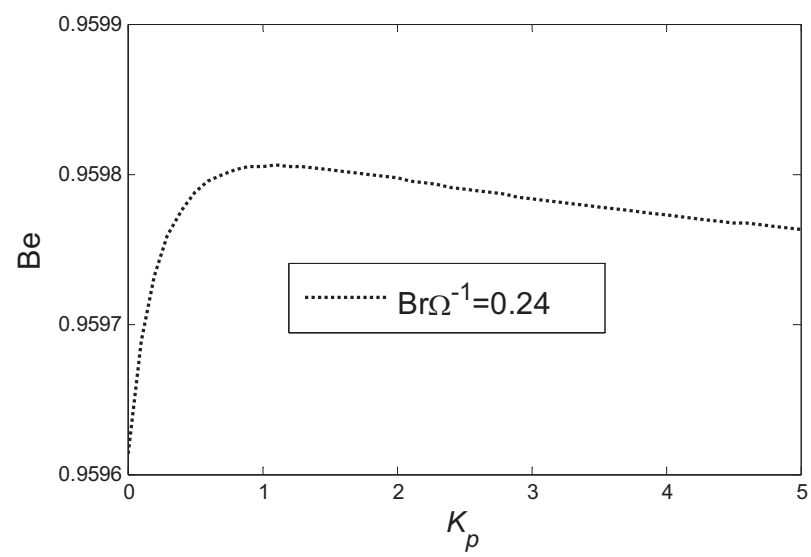

(b) 
Figure 9. (a) Entropy generation number and (b) Bejan number as a function of $K_{p}$ for various values of $\operatorname{Re}$ when $\mathrm{Br} \Omega^{-1}=0.2, f_{w p}=0.01, n=0.3, \mathrm{X}=0.3, \operatorname{Pr}=5, \mathrm{Ec}=0.1$, $M=0.1, k^{\prime}=0.01, m^{\prime}=100, \zeta=0.00001, \lambda=0.0001$.

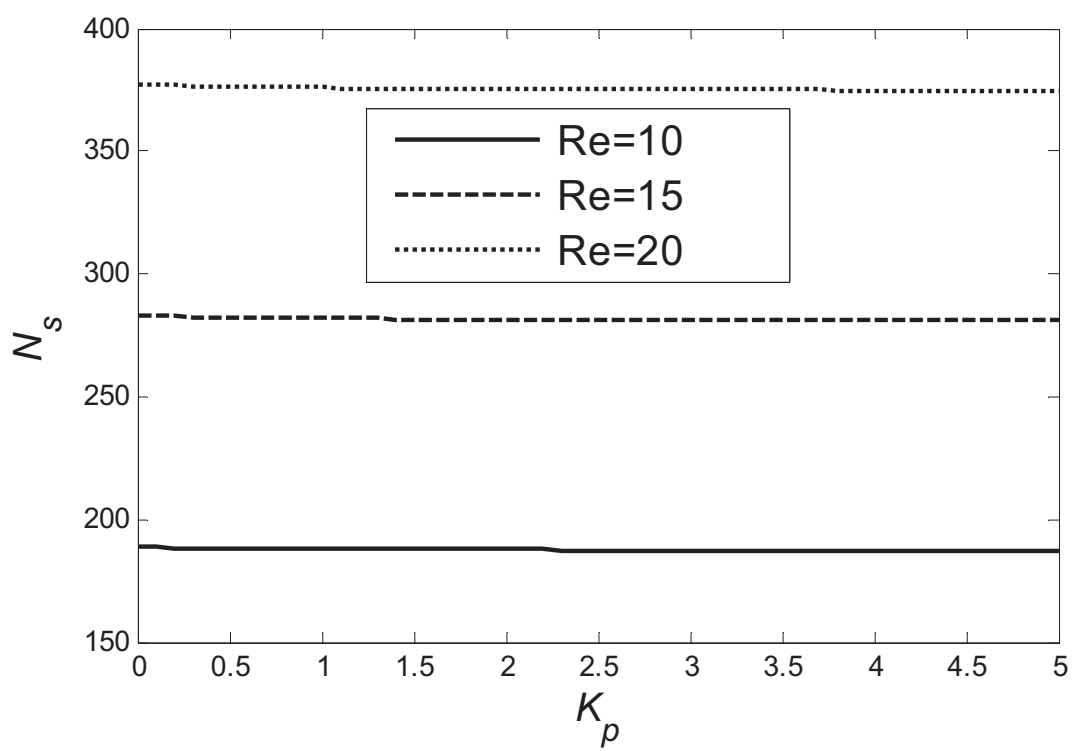

(a)

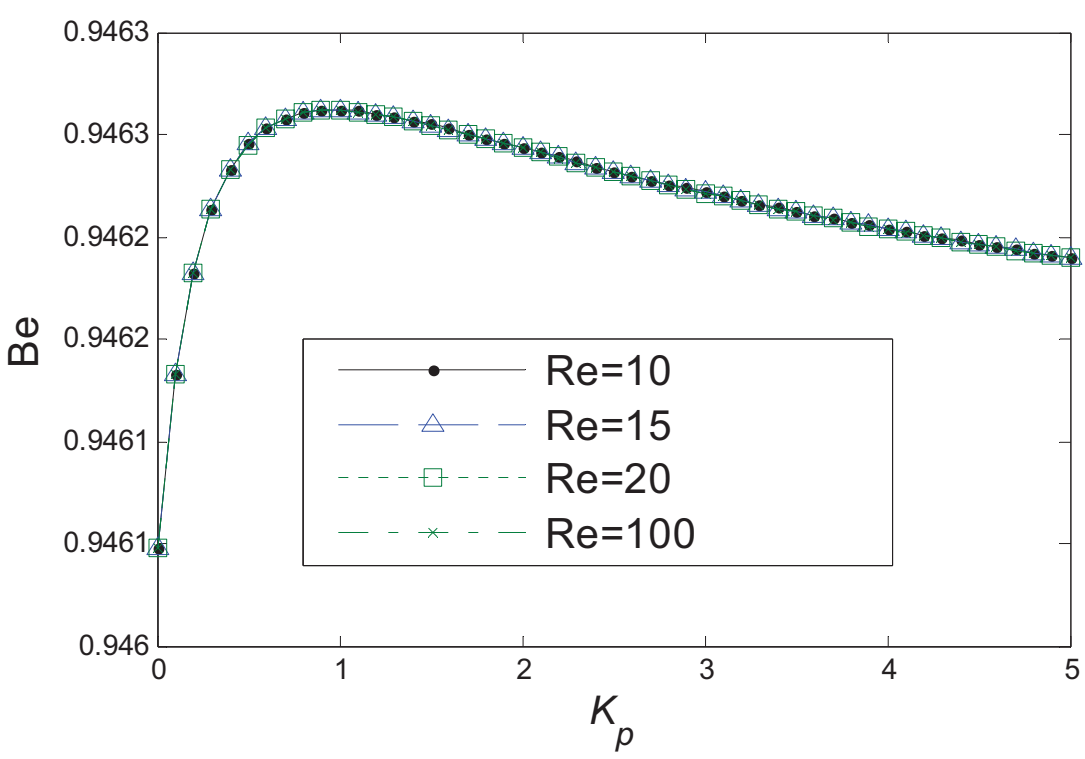

(b)

Figures $10 \mathrm{a}, \mathrm{b}$ show the combination effect of the suction/injection parameter and the slip coefficient on the entropy generation number and Bejan number, respectively. The entropy generation number is lower for a higher slip coefficient, whereby the presence of the suction creates entropy along the surface, with a noticeable opposite effect resulting from injection. Moreover, Bejan number decreases when $f_{w p}$ is increased for injection, increasing for suction. It is also evident that the heat transfer irreversibilities are more dominant in the case of suction, when compared to the injection. The effect of the surface temperature parameter on the entropy generation number and Bejan number is shown in Figures $11 \mathrm{a}, \mathrm{b}$ respectively, where it can be noted that an increase in the surface temperature parameter results in an increase in the both $N_{s}$ and $B e$ as the heat transfer irreversibility increases. 
Figure 10. (a) Entropy generation number and (b) Bejan number as a function of $K_{p}$ for various values of $f_{w p}$ when $\mathrm{Br} \Omega^{-1}=1, n=0.5, \mathrm{X}=0.3, \mathrm{Re}=10, \operatorname{Pr}=5, \mathrm{Ec}=0.1, M=0.1$, $k^{\prime}=0.2, m^{\prime}=100, \zeta=0.00001, \lambda=0.0001$.
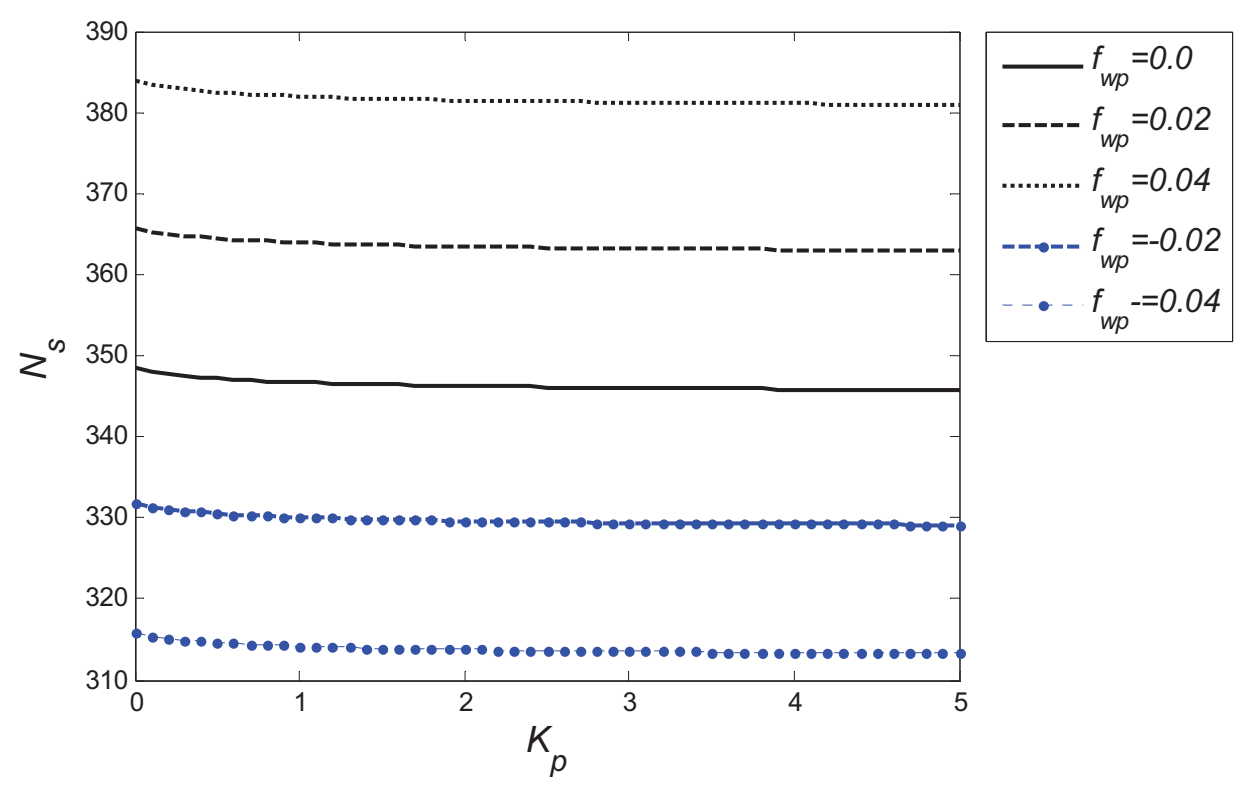

(a)
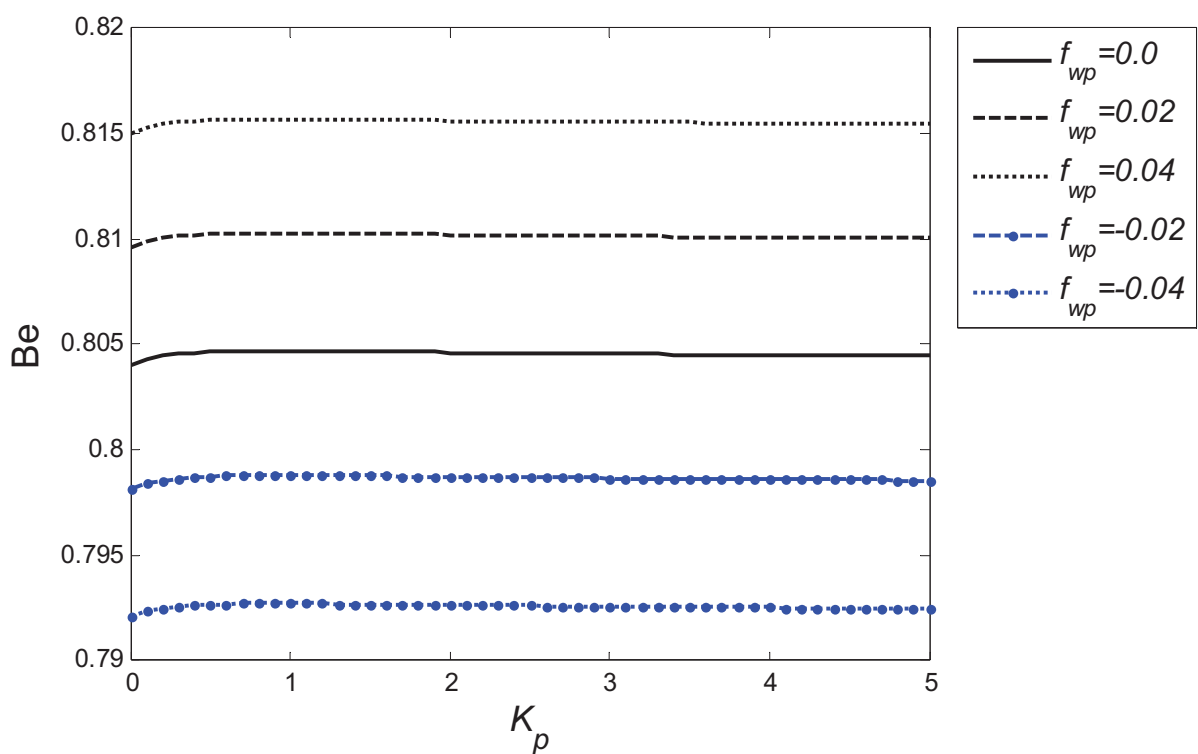

(b) 
Figure 11. (a) Entropy generation number and (b) Bejan number as a function of $K_{p}$ for various values of $k^{\prime}$ when $\mathrm{Br} \Omega^{-1}=1, f_{w p}=0.2, n=0.5, \mathrm{X}=0.3, \operatorname{Re}=10, \operatorname{Pr}=5, \mathrm{Ec}=0.1$, $M=0.1, m^{\prime}=100, \zeta=0.00001, \lambda=0.0001$.

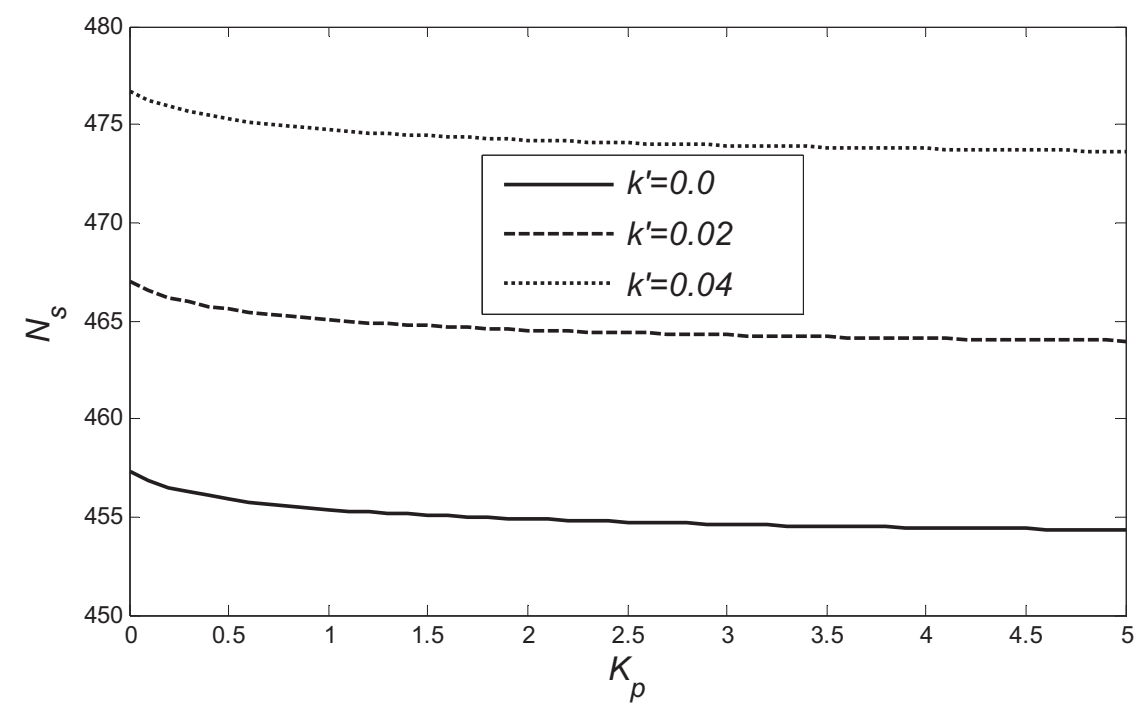

(a)

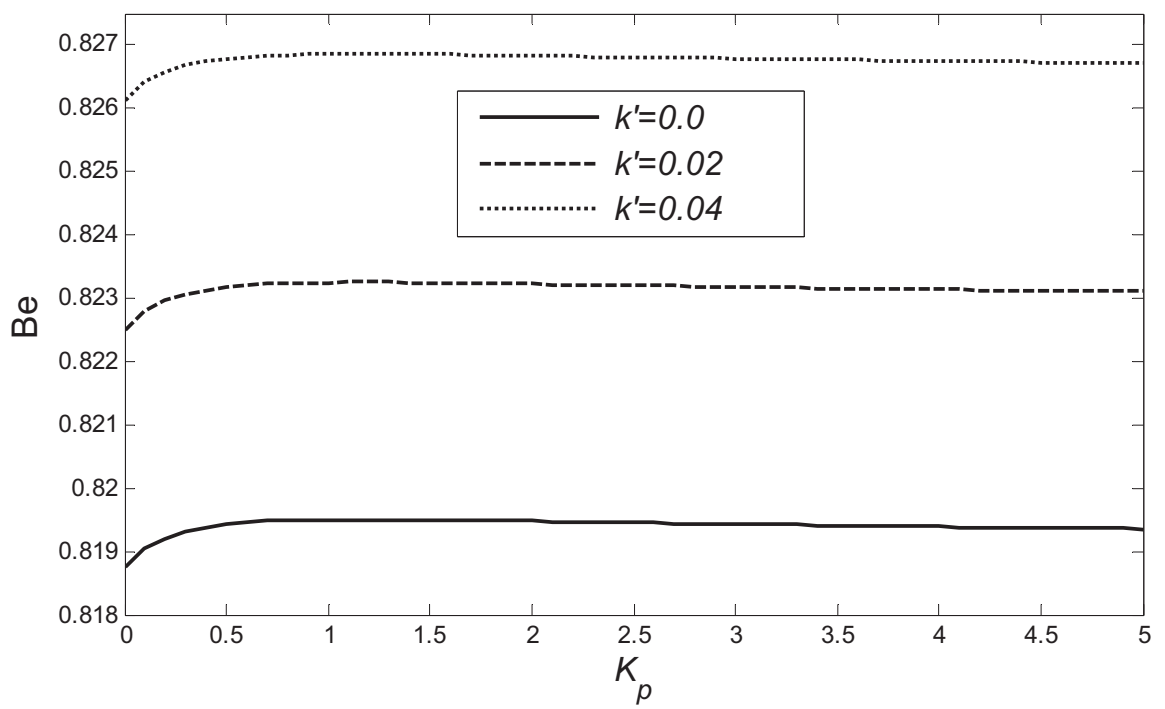

(b)

Figures 12a,b show the results of the change of the entropy generation number and Bejan number as a function of the change in the number of embedded open parallel microchannels, respectively. The interception point between the graphs determines different trends resulting from the larger slip coefficients, as compared to the smaller slip coefficients (before the interception point). As a greater surface area results in an increased surface friction due to a larger number of embedded microchannels, when the slip coefficient inside the microchannels is not sufficient, an increase in the number of microchannels tends to increase the entropy generation number, due to added surface friction. This phenomenon is much more pronounced when no-slip boundary condition $\left(K_{p}=0\right)$ is assumed inside the embedded microchannels, as no evident difference between slip-flow and no-slip behaviour is observed. Consequently, extra effort and cost associated with micromachining the surface to achieve a 
desired embedded microchannel surface cannot be warranted. However, for high values of the slip coefficient (after the interception point), an increase in the number of open parallel microchannels can effectively decrease the entropy generation number. Consequently, it is necessary to consider the projected values of the slip coefficients inside the microchannels required in order to establish an appropriate design of the open parallel microchannels embedded within the surface due to a reduction in the exergy losses. This can be effectively achieved by considering hydrophobic open microchannels with high slip coefficients. It is interesting to note that an increase in the number of microchannels causes an increase of the Bejan number's maximum value. Furthermore, at points to the left of the maximum, the slope of the tangent increases as the number of microchannels increases. This indicates that the heat transfer irreversibilities will be increasing at high $m^{\prime}$.

Figure 12. (a) Entropy generation number and (b) Bejan number as a function of $K_{p}$ for various values of $m^{\prime}$ when $\operatorname{Br} \Omega^{-1}=1, f_{w p}=0.2, n=0.5, \mathrm{X}=0.3, \operatorname{Re}=10, \operatorname{Pr}=5, \mathrm{Ec}=0.1$, $M=0.1, k^{\prime}=1, \zeta=0.00001, \lambda=0.0001$.

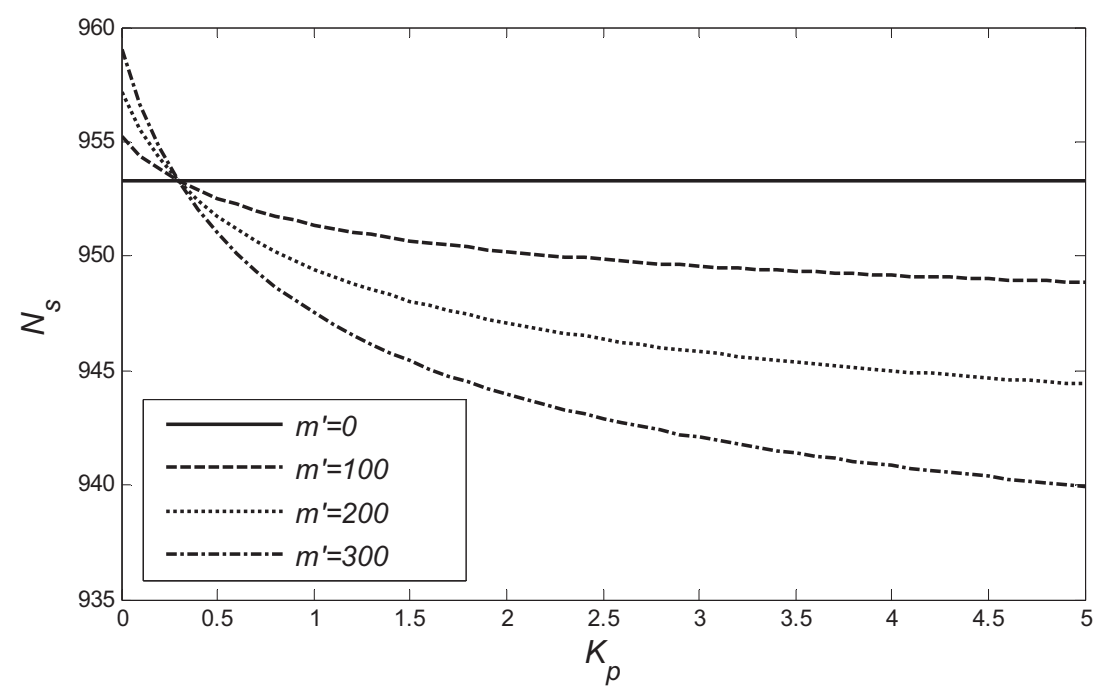

(a)

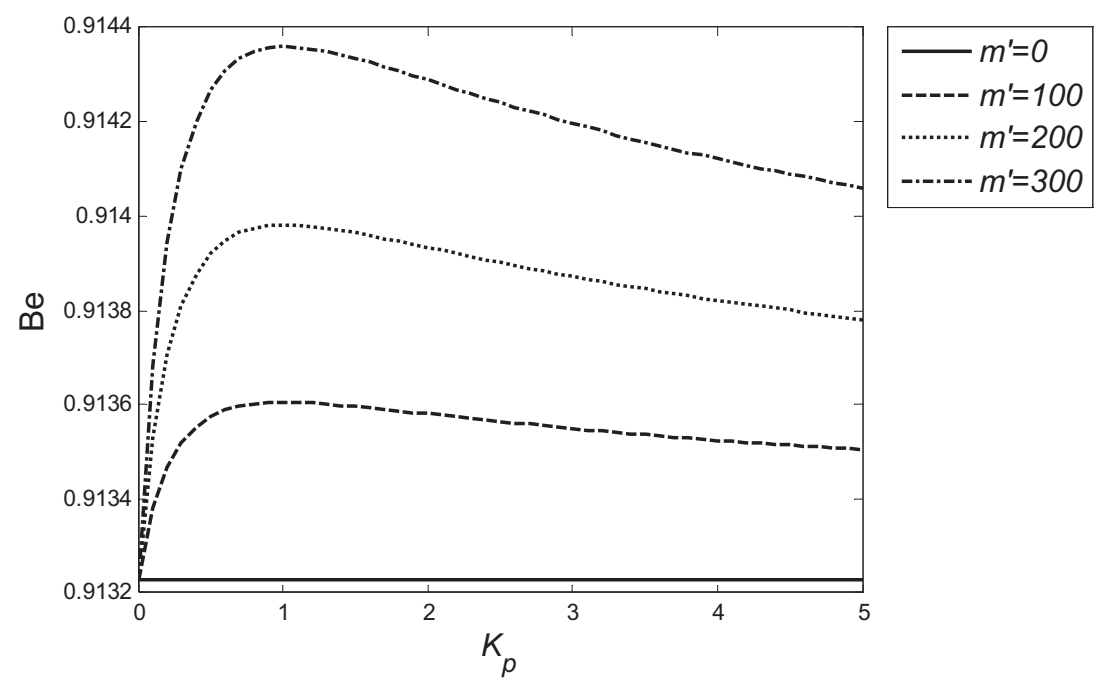

(b) 
Figure 13 shows change of the entropy generation number with varying surface non-dimensional geometric parameters and the slip coefficient. The entropy generation number shows an increase at higher microchannel depths, whereas it decreases at higher microchannel widths. This suggests that an increase in the width of the microchannels tends to enhance the slip effects along the width of the surface, causing the entropy generation number to decrease. The effect of the non-dimensional geometric parameters on the Bejan number is illustrated in Figure 14, which it increases with the increase in $\lambda$. It indicates that an increase in the width of the microchannels decreases the irreversibility distribution ratio with the increase of heat transfer irreversibilities. Further, it is noted that a decrease in the microchannel depth accompanies a slight rise in the Bejan number.

Figure 13. Effect of $\lambda$ and $\zeta$ on the entropy generation number when $\operatorname{Br} \Omega^{-1}=1, f_{w p}=0.2$, $n=0.5, \mathrm{X}=0.3, \mathrm{Re}=10, \operatorname{Pr}=5, \mathrm{Ec}=0.1, M=0.1, k^{\prime}=1$, and $m^{\prime}=100$.

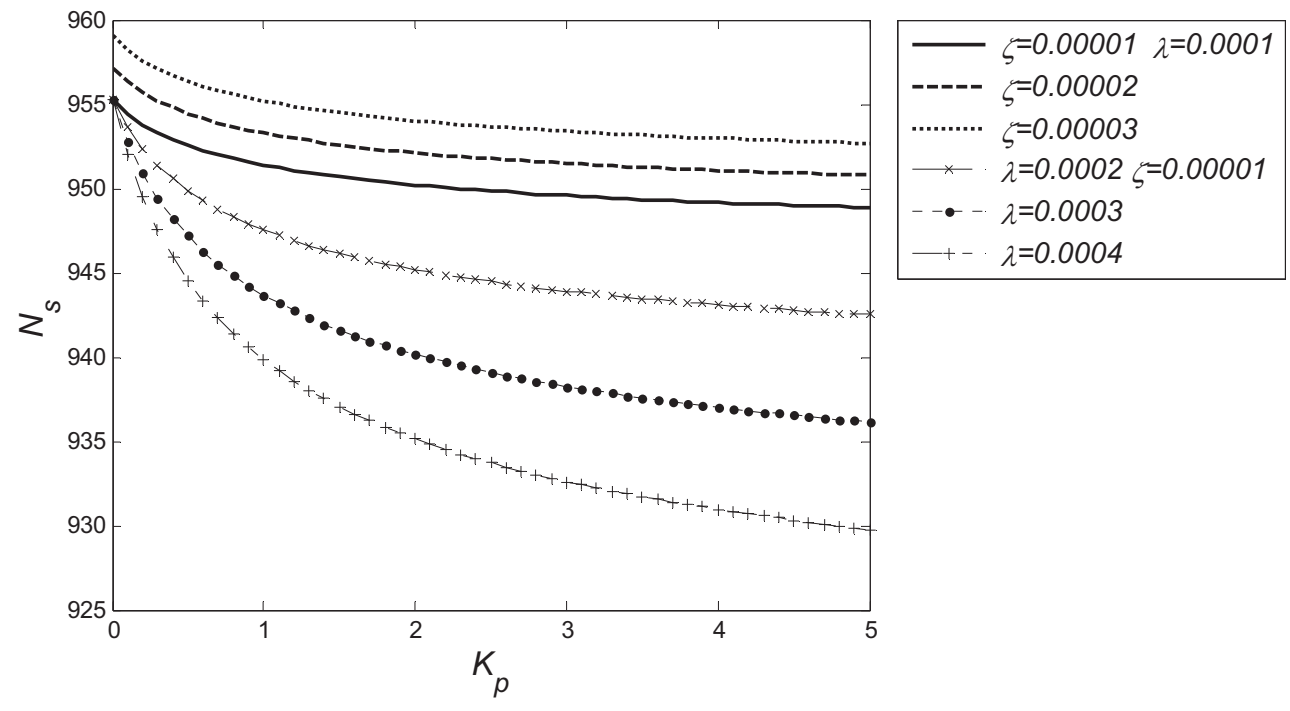

Figure 14. Effect of (a) $\lambda$ and (b) $\zeta$ on Bejan number when $\mathrm{Br}^{-1}=1, f_{w p}=0.2, n=0.5$, $\mathrm{X}=0.3, \operatorname{Re}=10, \operatorname{Pr}=5, \mathrm{Ec}=0.1, M=0.1, k^{\prime}=1$, and $m^{\prime}=100$.

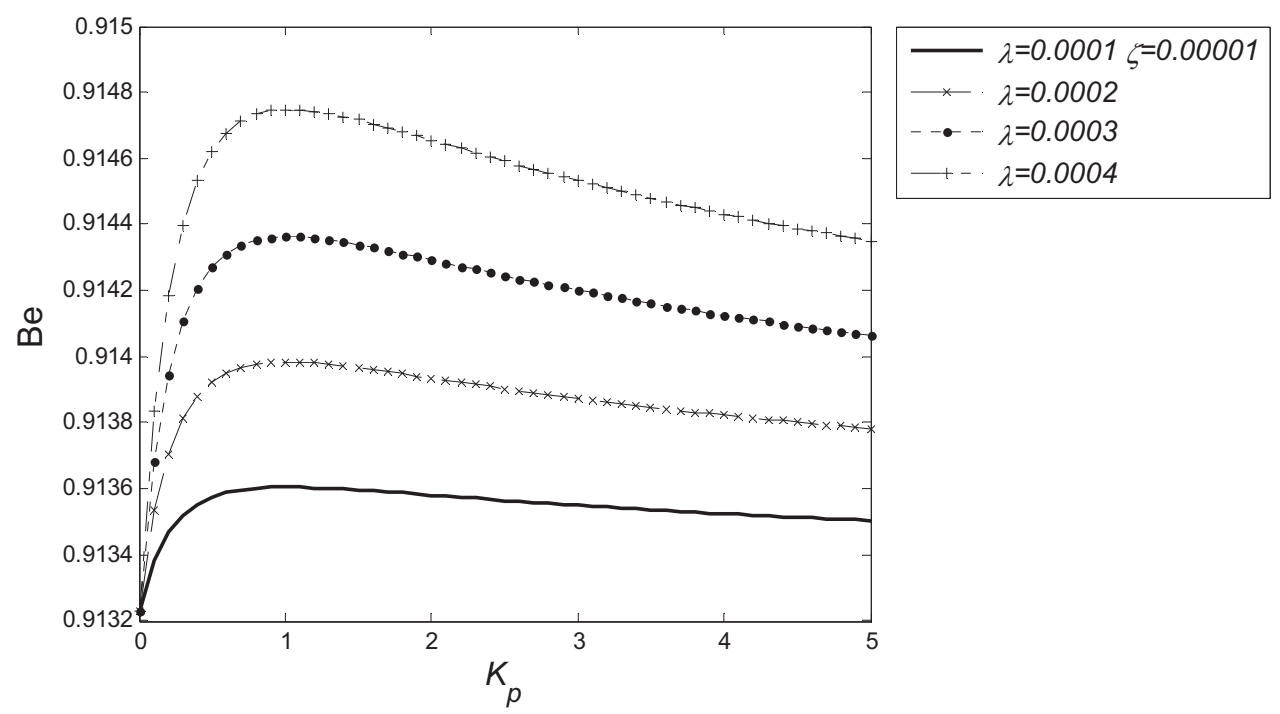

(a) 
Figure 14. Cont.

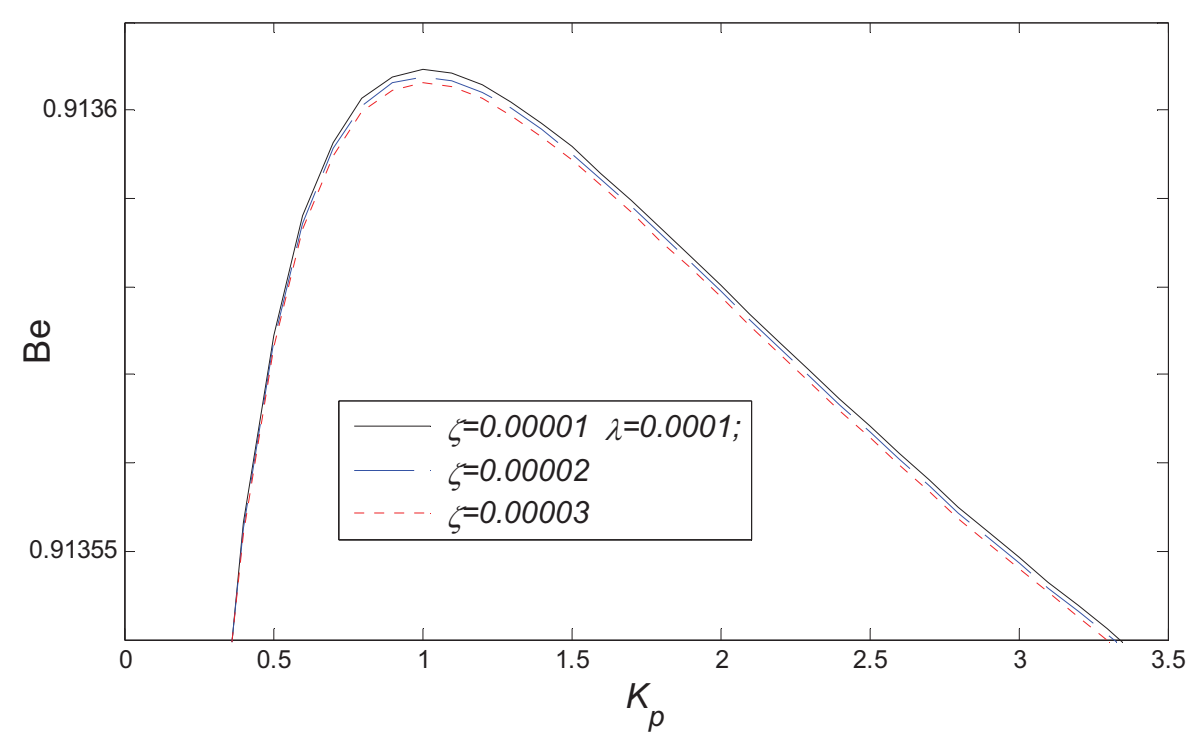

(b)

\section{Conclusions}

A new design of open parallel microchannels embedded within a permeable continuous moving surface due to the decrease in the exergy losses of magnetohydrodynamic (MHD) flow at prescribed surface temperature (PST) is evaluated. The entropy generation number is described by an integral of local entropy generation rate on the width of the surface, considering the effect of the embedded open parallel microchannels. Based on the results and discussions, the following conclusions can be reached:

- Joule heating decreases the heat transfer rate, in particular at high magnetic parameters.

- The entropy generation number $N_{s}$ decreases with the increase of injection, $K_{p}$ and $\lambda$, while it increases with the increase of suction, $\mathrm{Br} \Omega^{-1}, k^{\prime}$, Re and $\zeta$.

- The magnetic parameter can decrease the entropy generation number when the values of the heat transfer irreversibilities are much more significant compared to the fluid friction irreversibilities (at low $\mathrm{Br} \Omega^{-1}$ ).

- Bejan number, $B e$, increases with the increase of $k^{\prime}, m^{\prime}, \lambda$ and suction, while it decreases with the increase of $M, \operatorname{Br} \Omega^{-1}, \zeta$ and injection. The effect of $\operatorname{Re}$ on $B e$ is insignificant.

- There is a maximum value for $B e\left(K_{p}\right)$ that leads the slip coefficient to exhibit increasing (or decreasing) effect at different values.

- In hydrophobic open parallel microchannels with a high slip coefficient $K_{p}$ allow us to take advantage of an increase in the number of open parallel microchannels due to the reduction in the energy losses.

\section{Acknowledgments}

The authors would like to acknowledge the Ministry of Higher Education, Malaysia for sponsoring this work. They wish also to express their very sincerely thanks to the reviewers for their valuable comments and suggestions. 


\section{References}

1. Nisar, A.; Afzulpurkar, N.; Mahaisavariya, B.; Tuantranont, A. Mems-based micropumps in drug delivery and biomedical applications. Sens. Actuat. B Chem. 2008, 130, 917-942.

2. Chen, C.H.; Jang, L.S. Recent patents on micromixing technology and micromixers. Recent Patents on Mechanical Engineering 2009, 2, 240-247.

3. Capretto, L.; Cheng, W.; Hill, M.; Zhang, X. Micromixing within microfluidic devices. Top. Curr. Chem. 2011, 304, 27-68.

4. Kleinstreuer, C.; Li, J.; Koo, J. Microfluidics of nano-drug delivery. Int. J. Heat Mass Tran. 2008, $51,5590-5597$.

5. Avsec, J. Nanofluid and ferrofluid slip flow in rectangular and circular microchannels. In Proceedings of the 7th EUROMECH Solid Mechanics Conference, Lisbon, Portugal, 7-11 September, 2009.

6. Eijkel, J.C.T.; Berg, A. Nanofluidics: What is it and what can we expect from it? Microfluidics and Nanofluidics 2005, 1, 249-267.

7. Gad-el-Hak, M. The MEMS Handbook. CRC Press: Boca Raton, FL, USA, 2002.

8. Gad-el-Hak, M. MEMS: Applications. CRC Press: Boca Raton, FL, USA, 2005.

9. Javed, T.; Abbas, Z.; Sajid, M.; Ali, N. Heat transfer analysis for a hydromagnetic viscous fluid over a non-linear shrinking sheet. Int. J. Heat Mass Tran. 2011, 54, 2034-2042.

10. Hayat, T.; Javed, T.; Abbas, Z. Slip flow and heat transfer of a second grade fluid past a stretching sheet through a porous space. Int. J. Heat Mass Tran. 2008, 51, 4528-4534.

11. Fang, T.; Yao, S.; Zhang, J.; Aziz, A. Viscous flow over a shrinking sheet with a second order slip flow model. Comm. Nonlinear. Sci. Numer. Simulat. 2010, 15, 1831-1842.

12. Martin, M.J.; Boyd, I.D. Momentum and heat transfer in a laminar boundary layer with slip flow. J. Thermophys. Heat Tran. 2006, 20, 710-719.

13. Ariel, P.D. Axisymmetric flow due to a stretching sheet with partial slip. Comput. Math. Appl. 2007, 54, 1169-1183.

14. Bocquet, L.; Barrat, J.L. Flow boundary conditions from nano-to micro-scales. Soft Matter 2007, 3, 685-693.

15. Matthews, M.T.; Hill, J.M. Micro/nano thermal boundary layer equations with slip-creep-jump boundary conditions. IMA J. Appl. Math. 2007, 72, 894.

16. Yazdi, M.H.; Abdullah, S.; Hashim, I.; Zaharim, A.; Sopian, K. Friction and heat transfer in slip flow boundary layer at constant heat flux boundary conditions. In Proceedings of the 10th WSEAS International Conference on Mathematical Methods, Computational Techniques and Intelligent Systems, Corfu, Greece, 26-28 October, 2008; pp. 207-212.

17. Wang, C.Y. Analysis of viscous flow due to a stretching sheet with surface slip and suction. Nonlinear Anal. R. World Appl. 2009, 10, 375-380.

18. Hayat, T.; Qasim, M.; Mesloub, S. MHD flow and heat transfer over permeable stretching sheet with slip conditions. Int. J. Numer. Meth. Fluid. 2011, 66, 963-975.

19. Qasim, M.; Hayat, T.; Hendi, A. Effects of slip conditions on stretching flow with ohmic dissipation and thermal radiation. Heat Tran. Asian Res. 2011, 40, 641-654. 
20. Abbas, Z.; Wang, Y.; Hayat, T.; Oberlack, M. Slip effects and heat transfer analysis in a viscous fluid over an oscillatory stretching surface. Int. J. Numer. Meth. Fluid. 2009, 59, 443-458.

21. Yazdi, M.H.; Abdullah, S.; Hashim, I.; Sopian, K. Slip mhd flow over permeable stretching surface with chemical reaction. In Proceedings of the 17th Australasian Fluid Mechanics Conference, Auckland, New Zealand, 5-9 December 2010.

22. Fang, T.; Zhang, J.; Yao, S. Slip mhd viscous flow over a stretching sheet-an exact solution. Comm. Nonlinear Sci. Numer. Simulat. 2009, 14, 3731-3737.

23. Yazdi, M.H.; Abdullah, S.; Hashim, I.; Nopiah, Z.M.; Zaharim, A.; Sopian, K. Convective heat transfer of slip liquid flow past horizontal surface within the porous media at constant heat flux boundary conditions. In Proceedings of the American Conference on Applied Mathematics: Recent Advances in Applied Mathematics, Cambridge, MA, USA, 27-29 January, 2010; pp. 527-533.

24. Yazdi, M.H.; Abdullah, S.; Hashim, I.; Sopian, K. Slip mhd liquid flow and heat transfer over non-linear permeable stretching surface with chemical reaction. Int. J. Heat Mass Tran. 2011, 54, 3214-3225.

25. Wu, W.I.; Selvaganapathy, P.R.; Ching, C.Y. Transport of particles and microorganisms in microfluidic channels using rectified ac electro-osmotic flow. Biomicrofluidics 2011, 5, 013407.

26. Naterer, G.F. Surface micro-profiling for reduced energy dissipation and exergy loss in convective heat transfer. Microscale Thermophysical Engineering 2005, 9, 213-236.

27. Naterer, G.F. Reducing energy availability losses with open parallel microchannels embedded in a micropatterned surface. Int. J. Energ. Res. 2005, 29, 1215-1229.

28. Naterer, G.F.; Chomokovski, S. Entropy-based surface microprofiling for passive near-wall flow control. J. Micromech. Microeng. 2007, 17, 2138.

29. Naterer, G.F.; Glockner, P.S.; Thiele, D.; Chomokovski, S.; Venn, G.; Richardson, G. Surface micro-grooves for near-wall exergy and flow control: Application to aircraft intake de-icing. J. Micromech. Microeng. 2005, 15, 501.

30. Yazdi, M.H.; Abdullah, S.; Hashim, I.; Sopian, K.; Zaharim, A. Entropy generation analysis of liquid fluid past embedded open parallel microchannels within the surface. Eur. J. Sci. Res. 2009, $28,462-470$.

31. Al-Odat, M.Q.; Damseh, R.A.; Al-Nimr, M.A. Effect of magnetic field on entropy generation due to laminar forced convection past a horizontal flat plate. Entropy 2004, 6, 293-303.

32. Aïboud, S.; Saouli, S. Entropy analysis for viscoelastic magnetohydrodynamic flow over a stretching surface. Int. J. Non Lin. Mech. 2010, 45, 482-489.

33. Makinde, O.D. Second law analysis for variable viscosity hydromagnetic boundary layer flow with thermal radiation and newtonian heating. Entropy 2011, 13, 1446-1464.

34. Aïboud, S.; Saouli, S. Second law analysis of viscoelastic fluid over a stretching sheet subject to a transverse magnetic field with heat and mass transfer. Entropy 2010, 12, 1867-1884.

35. El Jery, A.; Hidouri, N.; Magherbi, M.; Brahim, A.B. Effect of an external oriented magnetic field on entropy generation in natural convection. Entropy 2010, 12, 1391-1417.

36. Makinde, O.D.; Bég, O.A. On inherent irreversibility in a reactive hydromagnetic channel flow. Int. J. Therm. Sci. 2010, 19, 72-79. 
37. Yazdi, M.H.; Abdullah, S.; Hashim, I.; Zaharim, A.; Sopian, K. Entropy generation analysis of the mhd flow over nonlinear permeable stretching sheet with partial slip. In Proceedings of the 6th IASME / WSEAS International Conference on Energy and Environment, EE'11, Cambridge, UK, 23-25 February 2011; pp. 292-297.

38. Rossow, V.J. On Flow of Electrically Conducting Fluids over A Flat Plate in the Presence of a Transverse Magnetic Field; National Advisory Committee for Aeronautics: Washington, DC, USA, 1957; NACA, TN 3971; Report no. 1358.

39. Lund, N.J.; Hendy, S.C. Effective slip length of nanoscale mixed-slip surfaces. ANZIAM J. 2009, 50, 381-394.

40. Niavarani, A.; Priezjev, N.V. The effective slip length and vortex formation in laminar flow over a rough surface. Phys. Fluid. 2009, 21, 052105.

41. Gad-el-Hak, M. The fluid mechanics of microdevices-the freeman scholar lecture. J. Fluids Eng. 1999, 121, 5-33.

42. Tretheway, D.C.; Meinhart, C.D. Apparent fluid slip at hydrophobic microchannel walls. Phys. Fluid. 2002, 14, L9.

43. Woods, L.C. The Thermodynamics of Fluid Systems; Oxford University Press: New York, NY, USA, 1985; Volume 2.

44. Sharp, K.V.; Adrian, R.J. Transition from laminar to turbulent flow in liquid filled microtubes. Exp. Fluid. 2004, 36, 741-747.

45. Makinde, O.D. Thermodynamic second law analysis for a gravity-driven variable viscosity liquid film along an inclined heated plate with convective cooling. J. Mech. Sci. Tech. 2010, 24, 899-908.

46. Kobo, N.S.; Makinde, O.D. Second law analysis for a variable viscosity reactive couette flow under arrhenius kinetics. Math. Probl. Eng. 2010, 2010, 1-15.

47. Reveillere, A.; Baytas, A.C. Minimum entropy generation for laminar boundary layer flow over a permeable plate. Int. J. Exergy 2010, 7, 164-177.

48. Ali, M. Heat transfer characteristics of a continuous stretching surface. Waerme und Stoffuebertragung 1994, 29, 227-234.

49. Ishak, A.; Nazar, R.; Pop, I. Heat transfer over an unsteady stretching permeable surface with prescribed wall temperature. Nonlinear Anal. R. World Appl. 2009, 10, 2909-2913.

(C) 2012 by the authors; licensee MDPI, Basel, Switzerland. This article is an open access article distributed under the terms and conditions of the Creative Commons Attribution license (http://creativecommons.org/licenses/by/3.0/). 